\title{
A review of tangle-veined flies (Nemestrinidae, Diptera) in Egypt
}

\author{
Arafa Elsayed El-Hashash ${ }^{1,2}$, Haitham Badrawy Mousa Badrawy ${ }^{3}$, \\ Ayman Mohyie-Eldin Ibrahim'
}

I Department of Taxonomy, Plant Protection Research Institute, Agricultural Research Centre, Dokki-Giza, Egypt 2 Plant Protection Department, Agricultural Faculty, Peoples' Friendship University of Russia, Moscow, Russia 3 Department of Entomology, Faculty of Science, Ain Shams University, Abbassia-Cairo, Egypt

Corresponding author: Arafa Elsayed El-Hashash (arafa.Elhashash@yahoo.com)

Academic editor:Torsten Dikow | Received 27 June 2021 | Accepted 8 October 2021 | Published 16 November 2021

http://zoobank.org/C91AFA18-87C8-444E-AB6C-85C747B3893B

Citation:El-Hashash AE, Badrawy HBM, Ibrahim AM-E (2021) A review of tangle-veined flies (Nemestrinidae, Diptera) in Egypt. ZooKeys 1071: 11-42. https://doi.org/10.3897/zookeys.1071.70743

\begin{abstract}
The Egyptian fauna of the genus Nemestrinus Latreille, 1802 is revised. In 1967, Steyskal and El-Bialy listed 12 species from the region, but only six species are now recognized. The primary type specimens of the species $N$. aegyptiacus (Wiedemann, 1828), N. rufipes (Olivier, 1810), and N. lateralis Wiedemann, 1828 ( $N$. lateralis being a synonym of $N$. rufipes) deposited in the Museum für Naturkunde, Berlin, Germany were examined. Two species (N. abdominalis Olivier, 1811 and $N$. fascifrons (Bigot, 1888) are placed as new synonyms of $N$. ater (Olivier, 1811), and $N$. ruficornis Macquart, 1840 is synonymized with N. rufipes (Olivier, 1811). Nemestrinus jullieni (Efflatoun, 1925) is confirmed as a synonym of N. aegyptiacus. Furthermore, three species (N. caucasicus Fischer, 1806, N. pallipes (Olivier, 1811), and N. persicus Lichtwardt, 1909) have been removed and are doubtful records from Egypt. A key to the species, lists of specimens examined, and Illustrations and distributions for each species are provided. The status of species of doubtful occurrence in Egypt is discussed.
\end{abstract}

\section{Keywords}

Distribution, Egypt, Nemestrinus, taxonomy 


\section{Introduction}

Nemestrinidae (tangle-veined flies) are a small dipteran family belonging to the suborder Brachycera-Orthorrhapha and occur all over the world, but are most abundant and diverse in the Palaearctic, Australian and Afrotropical Regions (Richter 1997; Pape et al. 2011). The family is classified into five subfamilies and comprises approximately 300 species worldwide in 23 genera, while 77 species in eight genera are known from the Palaearctic region (Richter 1997; Narchuk 2007; Papavero and Bernardi 2009; Pape et al. 2011).

The Nemestrininae comprise $c a .175$ species worldwide in six genera (Bernardi 1973; Papavero and Bernardi 2009). In the Palaearctic Region, the Nemestrininae currently include approximately 67 species in two genera (Nemestrinus Latreille, and Stenopteromyia Lichtwardt) according to the last published catalogue by Richter (1988).

The genus Nemestrinus was described by Latreille in 1802 based on specimens collected from Egypt and Syria. It comprises 66 species in the Palaearctic Region (Bernardi 1973; Richter 1988, 1997; Narchuk 2007) and is characterized by the wing venation: The apical part of the wing occasionally has supernumerary transverse veins, $R_{3}$ is present, $R_{3+4}$ and $R_{5}$ are free, $M_{1}$ and $M_{2}$ are free, the diagonal vein reaches the wing margin, and the proboscis is well developed and longer than the head. One of the important tools to separate nemestrinid species is the genitalia, composed of the well-developed hypandrium, partly fused with the gonocoxites with a linguiform apical projection bearing numerous hairs; and the elongate gonocoxal apodemes, which are sinuate and fused medially forming a dorsal bridge (Richter and Ovtshinnikova 1996; Richter 1997).

Nemestrinus is primarily distributed along the arid desert belt of the Palaearctic Region where several species occur in North Africa (Morocco, Algeria, Tunisia, Libya, and Egypt) and the Middle East (Arabia, Israel, Iran), east to Central Asia, as far as Mongolia and southern Russia, and in addition southern Europe (Bulgaria, Romania, Ukraine, France, Spain, and Turkey). The genus penetrates south into the Saharan part of the Afrotropical Region, being recorded from Sudan and Ethiopia (Bernardi 1973; Richter 1988, 1997). The type localities of seven species are situated in Egypt: $N$. abdominalis Olivier, 1811, N. aegyptiacus (Wiedemann, 1828), $N$. ater (Olivier, 1811), $N$. fasciatus (Olivier, 1811), N. reticulatus Latreille, 1802, N. ruficornis Macquart, 1840, and N. rufipes (Steyskal and El-Bialy 1967; Bernardi 1973; Richter 1988).

Two catalogues cover the nemestrinid fauna of Egypt: the monograph of Sack (1933) lists ten species and one variety [Nemestrellus abdominalis, N. ater, N. exalbidus (Lichtwardt, 1907), N. fascifrons, N. ruficornis, N. rufipes, Nemestrinus aegyptiacus, Ne. a. var. jullieni, Ne. persicus, Ne. reticulatus, and Rhynchocephalus fasciatus] in three genera (Nemestrellus, Nemestrinus, Rhynchocephalus), while Steyskal and El-Bialy (1967) list eleven species and one variety in the same three genera, adding $R$. caucasicus to Sack's monograph. There are also outdated works (e.g., Bequaert 1932, 1938) on the taxonomic status of the genus. Bernardi (1973) reviewed the world genera, and Richter (1988) presented the Palaearctic catalogue of Nemestrinidae; both listed ten species in Egypt, removing the same two species (Nemestrinus caucasicus and N. persicus) listed by Steyskal and El-Bialy (1967). Bernardi (1973) and Richter (1988) added N. pallipes that was not listed in Steyskal and El-Bialy (1967) as Egyptian species. 
There is no modern comprehensive work identifying and cataloguing the Egyptian nemestrinine fauna. The subfamily in Egypt has never been monographed, and the genus is very much in need of a modern revision. This study was undertaken to revise, update, and clarify the taxonomic status of the species of genus Nemestrinus Latreille in the Egyptian fauna.

\section{Materials and methods}

Specimens examined in this study are deposited in the following collections:

ASUC Entomology Department, Faculty of Science, Ain Shams University

AZUC Faculty of Agriculture, Alfieri, Al Azhar University

CUC Entomology Department, Faculty of Science, Cairo University

ESEC Entomological Society of Egypt

MAC Department of Taxonomy, Plant Protection Institute, Ministry of Agriculture NHMW Naturhistorisches Museum Wien, Austria

The Museum für Naturkunde, Germany, Berlin (ZMHB) is the depository of type specimens of $N$. aegyptiacus, $N$. rufipes, and $N$. lateralis Wiedemann, 1828 (the latter is a synonym of the second species). We obtained this information by personal communication with Mr. Sven Marotzke and Ms. Elena Grigoryeva.

We could not access the types of other species because some are missing, as in the Egyptian Society of Entomology, wherein type specimens of the species $N$. jullieni have apparently been destroyed, and it is not known where the other types are. We examined and revised the original descriptions of all Egyptian nemestrinid species.

The Smithsonian National Museum of Natural History (USNM) has specimens of Nemestrinidae from Egypt: $N$. abdominalis, $N$. aegyptiacus, and $N$. rufipes that have all been identified by Dr. Torsten Dikow using our key. Redescriptions are based on series of specimens of each of these species and body measurements include genitalia.

Morphological terms follow McAlpine et al. (1981), Richter and Ovtshinnikova (1996), Richter (1997) and Cumming and Wood (2017). Line drawings of body parts were made by using a stereomicroscope at a magnification of $40 \times$. We have access to the photographs by Ms. Elena Grigoryeva, Mr. Sven Marotzke, and Bernhard Schurian of the types that were downloaded at https://doi.org/10.7479/4wgc-dv22.

\section{Taxonomic account}

\section{Nemestrinus}

Nemestrinus Latreille, 1802: 437. Type species: Nemestrinus reticulatus Latreille, 1802: 437.

Rhynchocephalus Fischer, 1806: 219-220. 
Andrenomyia Rondani, 1850: 189.

Heminemestrinus Bequaert, 1932: 21.

Symmictoides Bequaert, 1932: 105.

Nemestrellus Sack, 1933: 7.

Nemestrina Rondani, 1850: 189, 197: incorrect subsequent spelling of Nemestrinus Latreille, 1802 or subsequent usage of Nemestrina Blanchard, 1845: 468.

Remarks. Currently there are six species the Egyptian fauna ( $N$. aegyptiacus, $N$. ater, $N$. exalbidus, $N$. fasciatus, N. reticulatus, and $N$. rufipes). The type specimens of $N$. jullieni deposited in ESEC have been destroyed by dermestid beetles and the types of the species $N$. aegyptiacus and $N$. rufipes and the type of latter's synonym $N$. lateralis are deposited in ZMHB.

Three species ( $N$. caucasicus $N$. pallipes, and $N$. persicus) have been treated as doubtful since there is no evidence of their occurrence in Egypt. This is based on their known distributions as listed in the world catalogue by Bernardi (1973), the Palaearctic catalogue by Richter (1988), and the Systema Dipterorum (Thompson and Pape 2021). Additionally, the type localities of $N$. caucasicus and $N$. persicus are in the Caucasus, Iran, and Jaffa (Israel) respectively, not in Egypt.

\section{Key to the Egyptian species of Nemestrinus}

1 Wing without supernumerary transverse veins (Fig. 65) ....................N. fasciatus

- Wing with supernumerary transverse veins, resulting in reticulate venation (Fig. 8) 2

2 Small cells extending forward posterior to R1 (Fig. 27); frons shiny black with a transverse white band. N. ater

- Small cells extending forward posterior to R2 (Fig. 18); frons entirely pollinose or with a shiny spot.

3 Small cells restricted between R2 and hind margin (Fig. 85); abdomen entirely black or grey with transverse black stripes ........................................................ 4

- Small cells restricted between R2 and M1 or M2 (Figs 46, 90); abdomen orange with a longitudinal black vitta (Figs 57,101$)$................................................. 5

4 Abdomen entirely black (Fig. 1); frons entirely pollinose (Figs 4, 5)

N. aegyptiacus

- Abdomen gray with incomplete transverse black stripes (Fig. 84); frons with a shiny spot (Figs 80, 81) N. reticulatus

5 Frons yellowish black; vertex black; venter of abdomen black with yellowish incisions N. pallipes

- Frons yellow or grey; vertex black or brown; venter of abdomen entirely orange or with black sides.

6 Frons yellow pollinose; tergum II with a transverse white band (Figs 2, 3, 91); venter of abdomen orange and black laterally.

- Frons grey pollinose; tergum II without a transverse white band (Fig. 47); venter of abdomen entirely orange. 


\section{Nemestrinus aegyptiacus Wiedemann, 1828}

Figures 1, 4-22

Nemestrinus aegyptiacus Wiedemann, 1828: 249.

Nemestrinus tripolitana Lichtwardt, 1907: 443.

Nemestrinus jullieni Efflatoun, 1925: 357.

Type material. Nemestrinus aegyptiacus: Syntype female, without date, Egypt (ZMHB) (pers. comm., Mr. Sven Marotzke). Nemestrinus jullieni: Type W. Hoff $29^{\circ} 53^{\prime} 02.6 " \mathrm{~N}$, $31^{\circ} 18^{\prime} 42.2^{\prime \prime E}, 15 . i i i .1922$, Helwan $29^{\circ} 50^{\prime} 37.6^{\prime \prime N}, 31^{\circ} 19^{\prime} 05.0^{\prime \prime} \mathrm{E}, 20 . i i i .1925$; Lec-

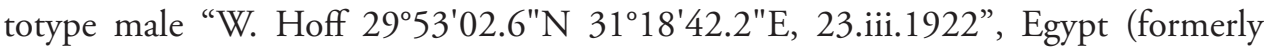
ESEC, destroyed by dermestid beetles).

Specimens examined. $N$. aegyptiacus: Burg El-Arab 3054'12.7"N, 29³3'13.7"E, 25.iii.1927 (1 f\#), 25.iii.1934 (1 m\#); Helwan 2950'37.6"N, 31 19'05.0"E, 17.iii.1934

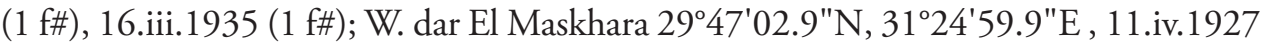

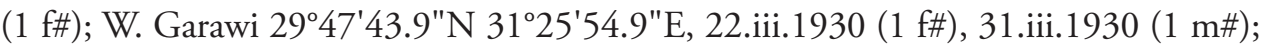

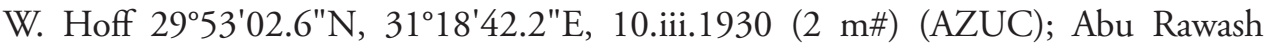
3004'30.7"N, 31¹1'59.7"E, 7.iii.1955 (9 m\#), 8.iii.1955 (3 m\# \& 1 f\#), 13.iii.1955 (4 m\# \& 3 f\#), 17.iii.1955 (5 m\# \& 4 f\#), 20.iii. 1955 (2 m\# \& 5 f\#); Giza 3000'40.0"N,

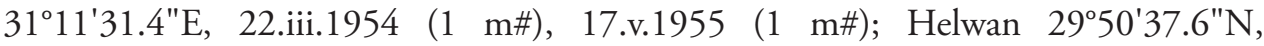
31 ${ }^{\circ} 19^{\prime} 05.0 " E$, 17.iii.1934 (1 m\#), 20.iii.1934 (1 m\#), 3.iv.1934 (1 f\#); Ogret ElSheik $29^{\circ} 52^{\prime} 50.1^{\prime \prime N}, 31^{\circ} 18^{\prime} 27.8^{\prime \prime E ~, ~ 25 . i i .1927 ~(1 ~ m \#) ; ~ W . ~ G a r a w i ~} 29^{\circ} 47^{\prime} 43.9^{\prime \prime} \mathrm{N}$ $31^{\circ} 25^{\prime} 54.9^{\prime \prime E}$, 25.iii.1932 (1 f\#); W. Rishrash $29^{\circ} 27^{\prime} 51^{\prime \prime N}, 31^{\circ} 22^{\prime} 2 " \mathrm{E}$, 29.iii.1935

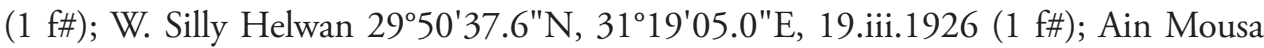
$29^{\circ} 52^{\prime} 22.0^{\prime \prime} \mathrm{N}, 32^{\circ} 39^{\prime} 00.7^{\prime \prime} \mathrm{E}, 16 . i i i .1925$ (2 f\#) (CUC); Asyut (Lentil) $27^{\circ} 23^{\prime} 00.0^{\prime \prime N}$,

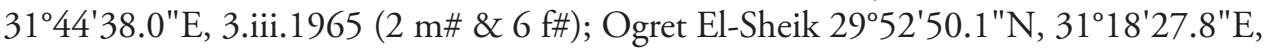
21.iii.1926 (2 f\#); Burg El-arab 3054'12.7"N, 29³3'13.7"E, 25.iii.1927 (1 m\#), 9.iii.1928 (1 m\#); Kafr Hakim 3004'39.7"N, 3106'46.3"E, $24 . i i i .1924$ (1 m\#); Gerga (Eg. Lupia) $26^{\circ} 20^{\prime} 23.2^{\prime \prime N}, 31^{\circ} 53^{\prime} 21.3^{\prime \prime E ~, ~ 2 . i v .1965 ~(2 ~ f \#) ; ~ W . ~ H o f f ~ 2953 ' 02.6 " N, ~}$ $31^{\circ} 18^{\prime} 42.2^{\prime \prime E}, 21 . i i i .1922$ (1 f\#), 22.iii.1927 (1 m\#); W. Morrah 22²'39.1"N,

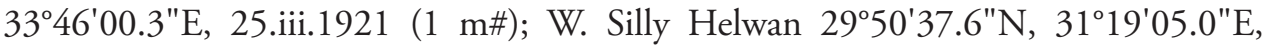

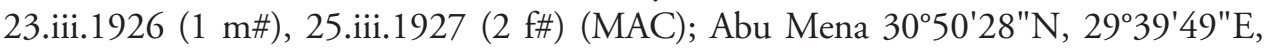

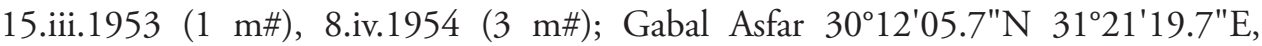
9.iii.1951 (1 f\#), 19.iii.1951 (1 f\#); Kerdasa 3001'32.1"N, 3106'27.5"E, 14.iv. 1951 (1 f\#), 20.iii.1952 (1 f\#) (ASUC); (1 m\#), without data (NHMW) sent by Dr. Peter Sehnal; Egypt (1 f\#), without date, (ZMHB) sent by Mr. Sven Marotzke and Bernhard Schurian; Cairo, Shoubra, 304'27.1632"N, 31ํ1'53.9844"E, 28.iii.1921 (1 m\#), specimen number USNMENT01371555 (USNM) (identified by Dr. Torsten Dikow).

Specimens previously identified as N. julieni: Abu Rawash $30^{\circ} 04^{\prime} 30.7^{\prime \prime} \mathrm{N}$, 31 ${ }^{\circ} 11^{\prime} 59.7^{\prime \prime E}$, 7.iii.1955 (1 m\#), 8.iii.1955 ( $1 \mathrm{m \# ),} \mathrm{13.iii.1955(1} \mathrm{m \# );} \mathrm{Burg} \mathrm{El-Arab}$ $30^{\circ} 54^{\prime} 12.7^{\prime \prime} \mathrm{N}, 29^{\circ} 33^{\prime} 13.7^{\prime \prime E}, 25 . i i i .1934$ (4 m\# \& 4 f\#); W. Garawi 2947'43.9"N, $31^{\circ} 25^{\prime} 54.9^{\prime \prime E}, 25 . i i i .1932$ (2 f\#), 21.iii.1930 (1 f\#); W. Hoff 2953'02.6"N,

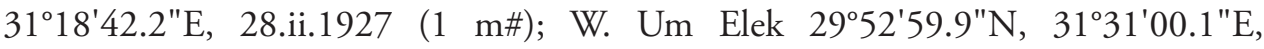
21.iii.1924 (1 f\#) (CUC); Ain Mousa 2952'22.0"N, 32³9'00.7"E , 16.iii.1925 (2 


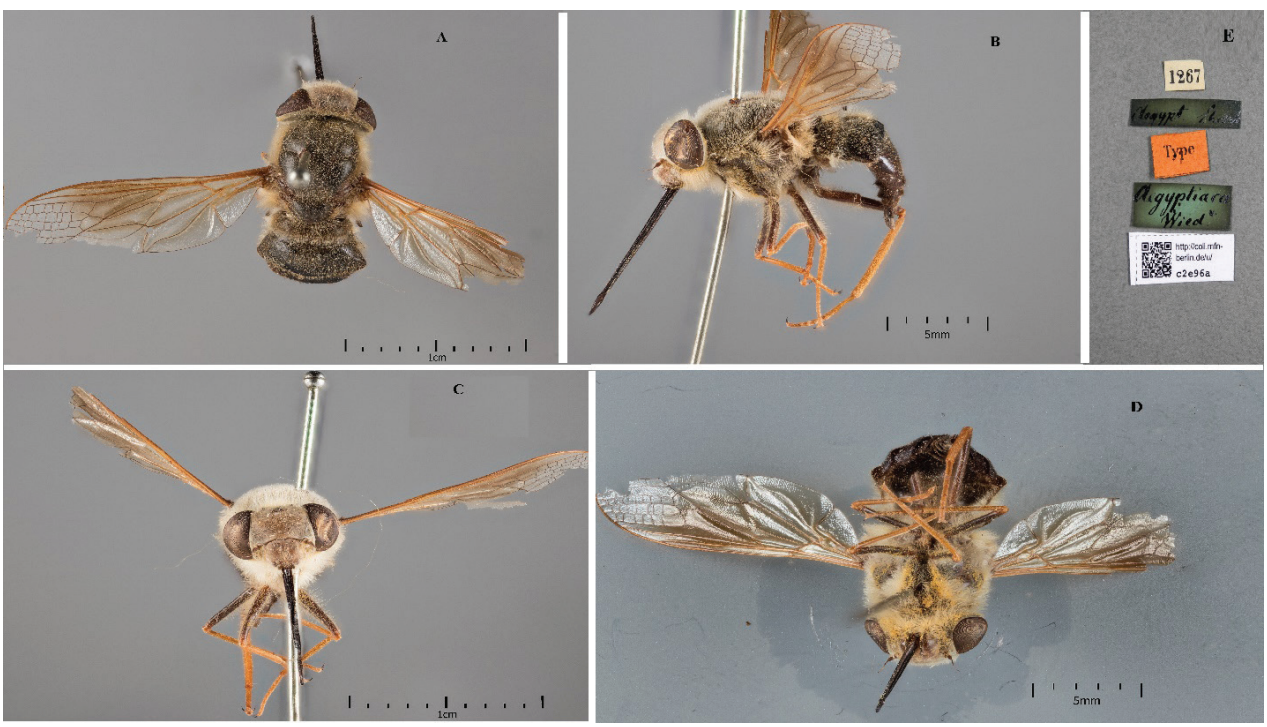

Figure I. Nemestrinus aegyptiacus, female syntype A dorsal view $\mathbf{B}$ lateral view $\mathbf{C}$ frontal view $\mathbf{D}$ ventral view E labels (ZMHB).

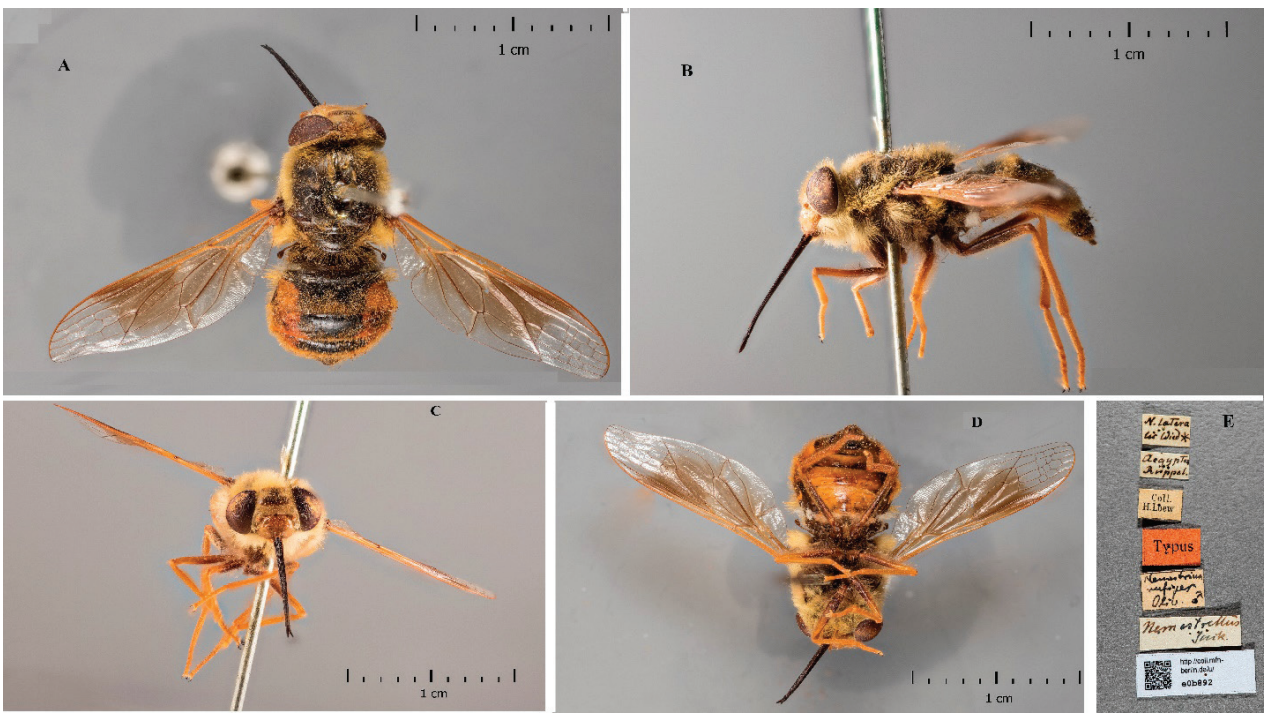

Figure 2. Nemestrinus rufipes, male type A dorsal view B lateral view C frontal view D ventral view E labels (ZMHB).

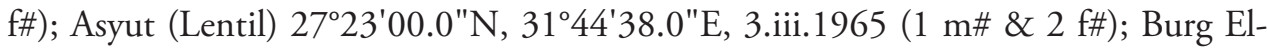
Arab 3054'12.7"N, 29³3'13.7"E, 14.iv.1920 (1 m\#), 16.ii.1922 (1 f\#),12.iv.1923 (1 f\#),11.iv.1925 (1 m\#), 18.iv.1925 (1 f\#); Kafr Hakim 3004'39.7"N, 3106'46.3"E, 20.iv.1925 (1 f\#); W. Garawi 2947'43.9"N, 31²5'54.9"E, $14 . i v .1928$ (1 f\#); W. Mor- 

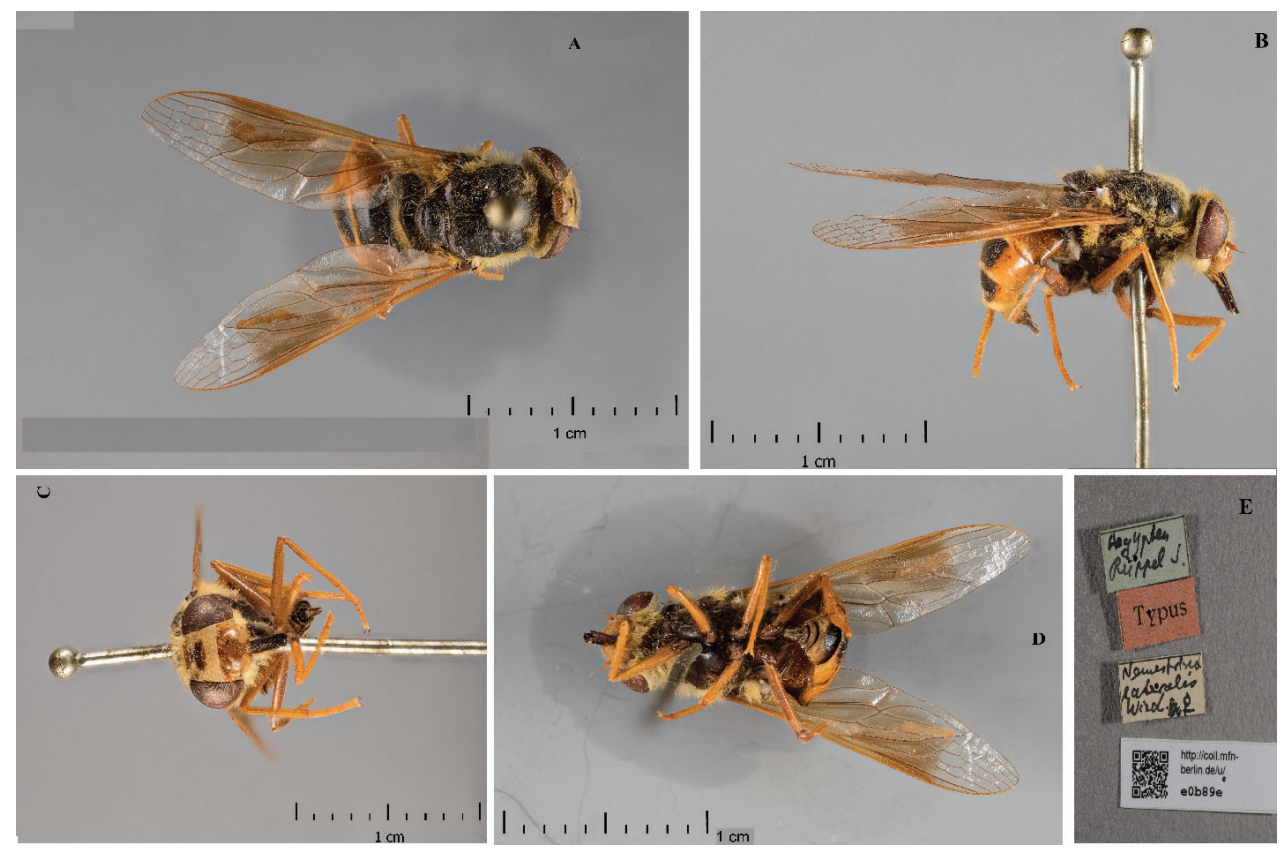

Figure 3. Nemestrinus lateralis (synonym), female syntype $\mathbf{A}$ dorsal view $\mathbf{B}$ lateral view $\mathbf{C}$ frontal view D Ventral View E Labels.

rah $22^{\circ} 22^{\prime} 39.1^{\prime \prime N}, 33^{\circ} 46^{\prime} 00.3^{\prime \prime E}, 25 . i i i .1927$ (1 f\#), W. Silly Helwan 2950'37.6"N, 31 19'05.0"E, 19.iii.1926 (2 f\#), 22.iii.1926 (1 f\#), W. Um Elek 2952'59.9” N,

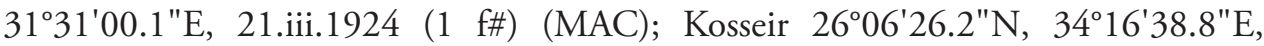

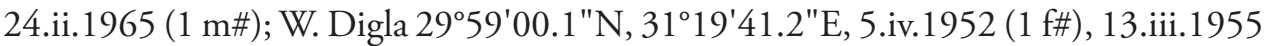
(1 m\#); W. Natroun 30²5'58.2"N, 30¹4'39.2"E, 13.iii.1955 (3 f\# \& 1 m\#), (2 m\#

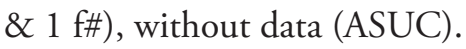

Diagnosis. Frons and face entirely yellow or grayish pollinosity; thorax completely shiny black with yellowish hairs; wing with many small cells restricted between R2 and hind margin; abdomen entirely black with short erect hairs. Male genitalia with only outer gonocoxal process; gonocoxal apodemes long, narrow, sinuate, fused medially and forming a narrow dorsal bridge; gonostyli wider than gonocoxal processes, ventrally with a cleft and small projection. Aedeagal complex with tapered aedeagus and lateral parameres, which are usually separated apically, fused with basal part of the aedeagus; parameral apodeme rather long; ejaculatory apodeme long and broad.

Redescription. Length: male body 14-17 mm, wing 13.5-15 mm. Female body 14-20 mm, wing 13.5-16.5 mm. Head wider than thorax; frons with yellow or grayish yellow pollinosity, with rather long hairs, at antennal elevation frons wide but narrowing toward vertex; face relatively shorter than high, with dull pollinosity, its hairs similar to those of the frons (Figs 4-6, 14-16); antenna entirely blackish brown to black, scape and pedicel with long hairs (Figs 7, 17). Thorax shiny black; mesonotum with dense pale yellow hairs but rather long; pleurae with tuft-like hairs. Leg hairy, 


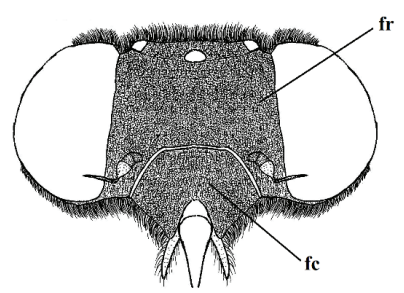

4

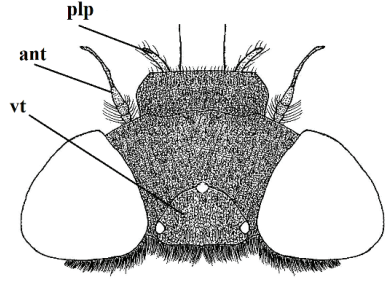

5

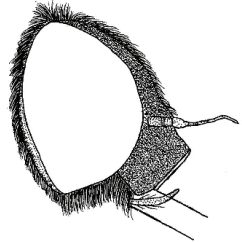

6

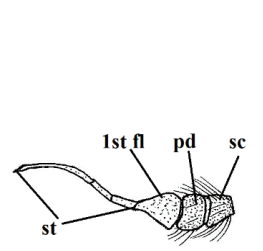

7

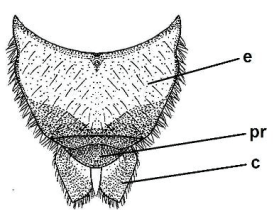

10

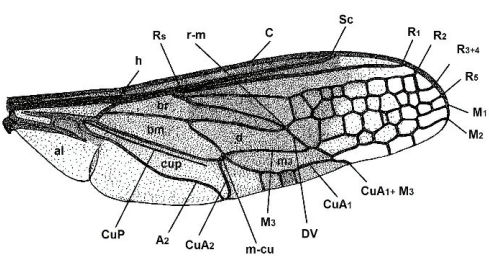

8

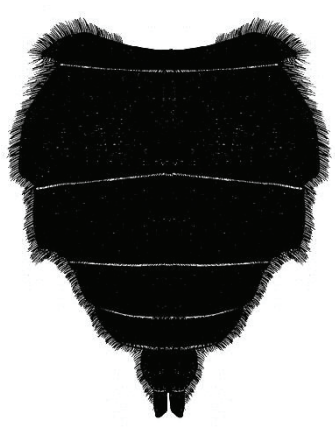

9

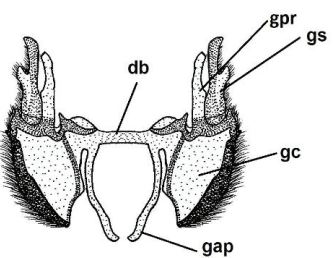

11

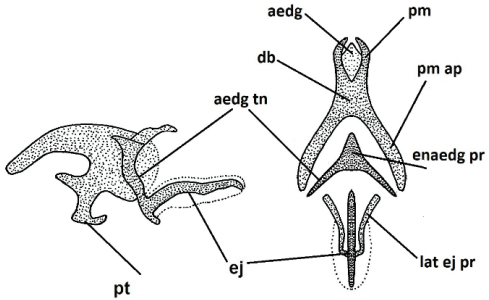

12

13

Figures 4-13. Male of Nemestrinus aegyptiacus, head, frontal (4), head, dorsal (5), head, lateral (6), antenna (7), wing (8), abdomen (9). 10-13 male genitalia: epandrium, proctiger, and cerci $(\mathbf{1 0})$, gonocoxite with gonostylus, ventral (I I), aedeagal complex, lateral (I 2) and dorsal (I3). Abbreviations: aedg. aedeagus, aec. aedeagal complex, aedg tn. aedeagal tine A2. anal vein, al. alula, ant. Antenna, bm. basal medial cell, br. basal radial cell, c. cerci, C. costa, CuA1,2, CuP. cubital veins, d. discal cell, db. dorsal bridge, DV. diagonal vein, e. epandrium, ej. ejaculatory apodeme, enaedg pr. endoaedeagal process, fc. Face, 1 st fl. first flagellomere, fr. Frons, gap. gonocoxal apodeme, gc. gonocoxite, gpr. gonocoxal process, gs. gonostylus, h. hypandrium, h. humeral cross vein, lat ej pr. lateral ejaculatory process, $\mathrm{m} 3$. third medial cell, pm. parameres, pm ap. parameral apodeme, pr. proctiger, pt. phallic plate, M1, M2, M3. medial veins, m-cu. cross vein between medial and cubital veins, pd, pedicel, plp. Palpus, R1, R2, R3+4, R5, Rs. radial veins, r-m. cross vein between redial and medial veins, Sc. subcostal vein, sc. Scape, st. stylus, vt. vertex.

yellowish brown with black femora. Wing smoky brown, except apex and posterapical margin clear and transparent (Figs 8, 18). The differences in cell number and structure on the wing is continuous variation and inconsistently different between 


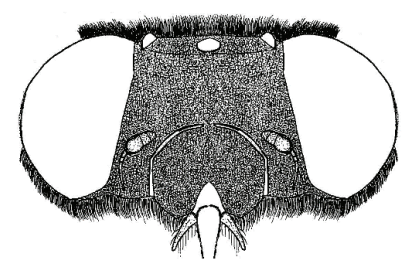

14

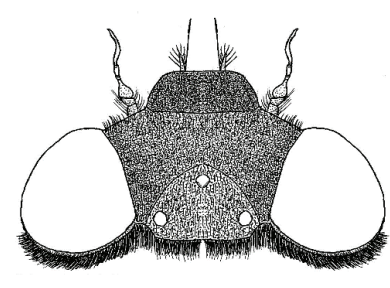

15

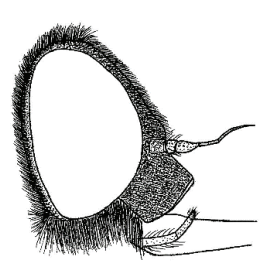

16

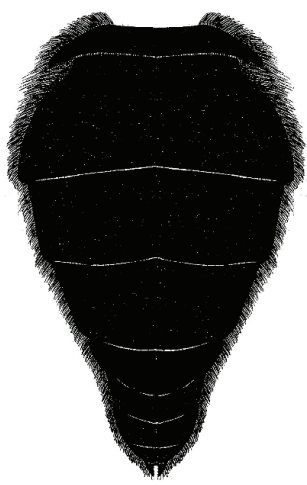

19
17

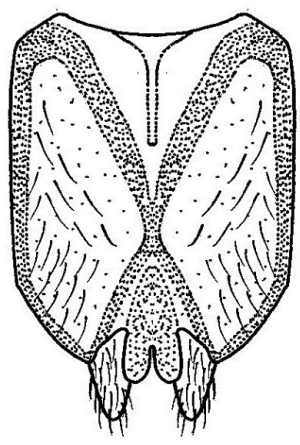

20

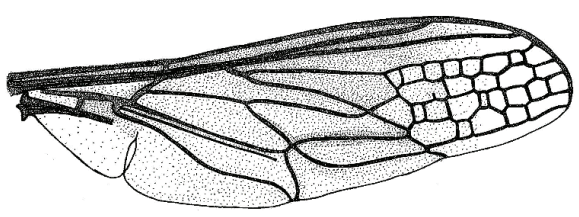

18

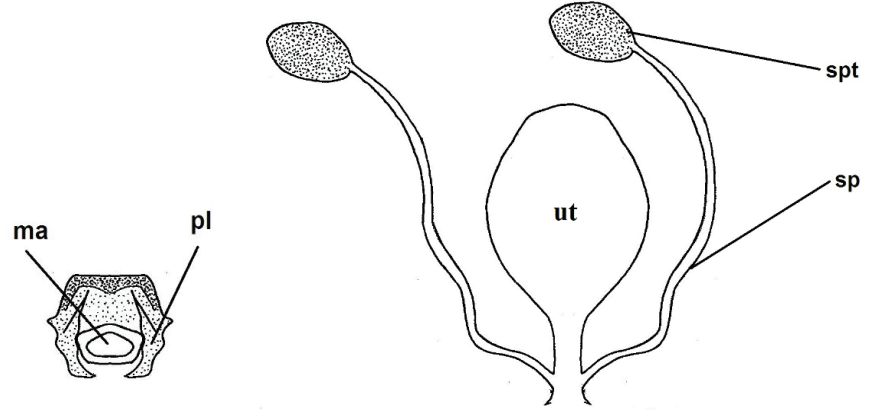

21

22

Figures 14-22. Female of Nemestrinus aegyptiacus, head, frontal (14), head, dorsal (I5), head, lateral (16), antenna (17), wing ( 1 8), and abdomen (19). 20-22 female genitalia: subgenital plate (20), genital furca (2I), and spermathecae (22). Abbreviations: ma. median aperture, pl. posterolateral projection, sp. spermatheca, spd. spermathecal duct, ut. uterus.

males and females. Abdomen entirely shiny black, covered with yellowish pubescence except the venter is blackish, which is rather short and erect, excluding dorsal side of two basal segments and on lateral margins of second segment where it is much longer and tufted, also on hind margin of each segment appear as narrow light bands (Figs 9, 19). Male genitalia with only outer gonocoxal process; gonocoxal apodemes long, nar- 
row, sinuate, fused medially, and forming a narrow dorsal bridge; gonostyli wider than gonocoxal processes, ventrally with a cleft and small projection (Figs 10,11). Aedeagal complex with tapered aedeagus and lateral parameres, which are usually separated apically, fused with basal part of aedeagus; parameral apodeme rather long; ejaculatory apodeme long and broad (Figs 12, 13). Female genitalia with subgenital plate rectangular with two hairy lobes (Fig. 20); genital furca free, narrow, with broadened ends of posterior projections, bent medially; median aperture of genital furca nearly triangular (Fig. 21); uterus rounded, with two narrow and rather long spermathecae (Fig. 22).

Local distribution. Coastal strip, Lower Nile.

Geographical distribution. Algeria, Egypt, Libya, Morocco, Italy (Sicily), and Tunisia (Sack 1933; Bernardi, 1973; Richter 1988).

Remarks. After examining the female type specimen of Nemestrinus aegyptiacus (Fig. 1) and comparing it with a large series of specimens identified as Nemestrinus jullieni Efflatoun (some specimens were by seen by him but it is not clear who determined the identification), we confirm this identification, and it is clear that both are the same species. Hence, $N$. julieni is placed as a synonym instead of a subspecies based on examination of the series of specimens and dissections of genitalia of both $N$. aegyptiacus and $N$. julieni and the female type specimen of $N$. aegyptiacus, and comparisons with the genitalia figures of Bernardi’s (1973: figs 54-56) N. aegyptiacus.

\section{Nemestrinus ater Olivier, 1811}

Figures 23-41

Nemestrinus ater Olivier, 1811: 171.

Nemestrinus abdominalis Olivier, 1811: 171. Syn. nov.

Nemestrinus nigra Wiedemann, 1828: 560.

Nemestrinus osiris Wiedemann, 1828: 561.

Nemestrina fascifrons Bigot, 1888: 8. Syn. nov.

\section{Type locality. Egypt.}

Specimens examined. Abu Rowash 3004'30.7"N, 31 $11^{\circ} 59.7^{\prime \prime E}$, 24.ii.1926

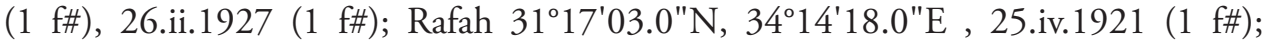
W. Garawi $29^{\circ} 47^{\prime} 43.9^{\prime \prime N}, 31^{\circ} 25^{\prime} 54.9^{\prime \prime E}, 31 . i i i .1930$ (1 f\#) (AZUC); W. Garawi $29^{\circ} 47^{\prime} 43.9^{\prime \prime} \mathrm{N}, 31^{\circ} 25^{\prime} 54.9^{\prime \prime} \mathrm{E}, 22 . i i i .1930$ (1 f\#), 31.iii.1930 (1 f\#) (CUC); Abu Qir 31 $18^{\prime} 42.4^{\prime \prime N}$, 3003'37.3"E, 26.iii.1915 (1 f\#); (Noaman Bey) Alexandria $31^{\circ} 10^{\prime} 03.5^{\prime \prime} \mathrm{N}, 29^{\circ} 51^{\prime} 56.2^{\prime \prime} \mathrm{E}$, (1 f\#, without date); Dekheila $31^{\circ} 07^{\prime} 32.0^{\prime \prime} \mathrm{N}$,

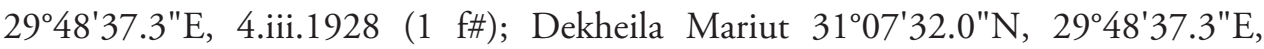
24.v.1925 (1 f\#); Burg El-Arab 3054'12.7"N, 29³3'13.7"E, 19.iv.1928 (1 f\#); Ga-

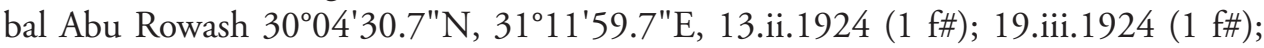
Kafr Hakim 3004'39.7"N, 3106'46.3"E, 24.iii.1925 (1 f\#), 20. iii. 1926 (1 f\#); Man- 


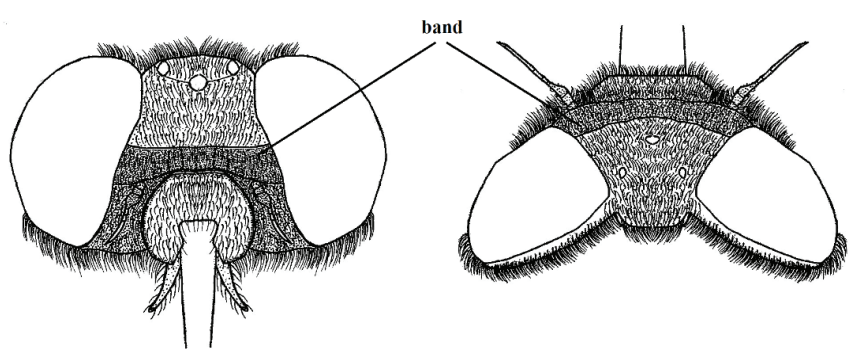

23
24

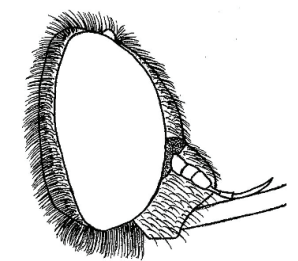

25

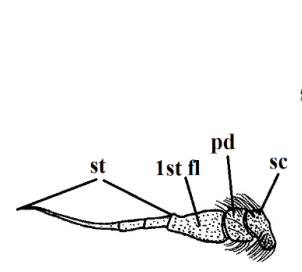

26

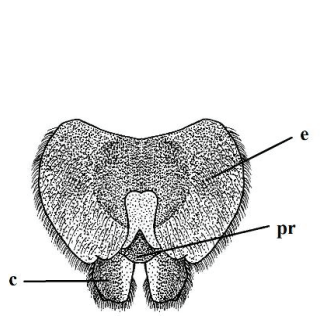

29

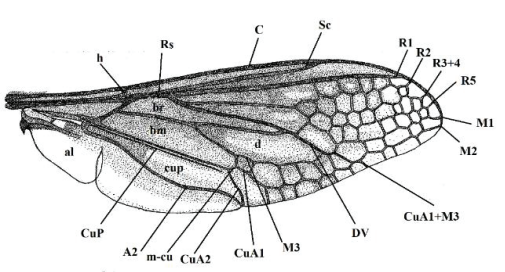

27

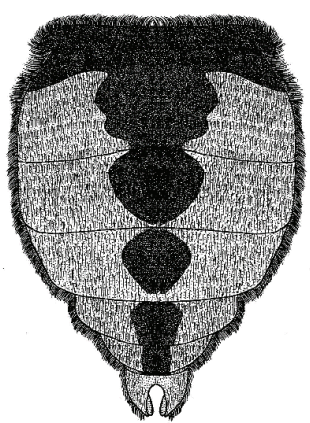

28

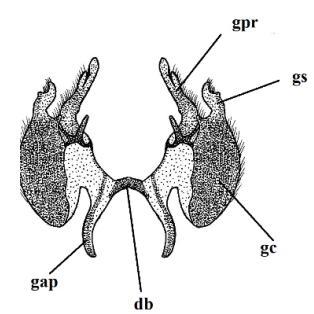

30

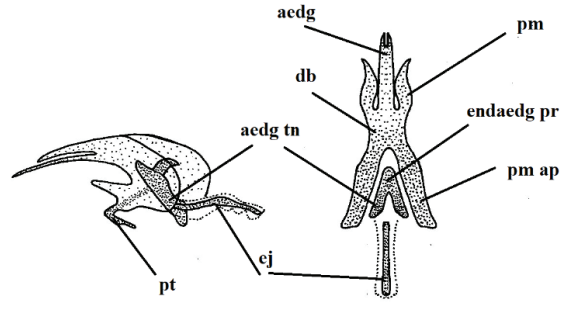

31

32

Figures 23-32. Male of Nemestrinus ater, head, frontal (23), head, dorsal (24), head, lateral (25), antenna (26), wing (27), and abdomen (28). 29-32 male genitalia: epandrium, proctiger, and cerci (29), gonocoxite with gonostylus, ventral (30), aedeagal complex, lateral (3I) and dorsal (32). Abbreviations: aedg. aedeagus, aec. aedeagal complex, aedg tn. aedeagal tine A2. anal vein, al. alula, bm. basal medial cell, br. basal radial cell, c. cerci, C. costa, CuA1,2, CuP. cubital veins, d. discal cell, db. dorsal bridge, DV. diagonal vein, e. epandrium, ej. ejaculatory apodeme, endaedg pr. endoaedeagal process, 1 st fl. first flagellomere, gap. gonocoxal apodeme, gc. gonocoxite, gpr. gonocoxal process, gs. gonostylus, h. hypandrium, h. humeral cross vein, lat ej pr. lateral ejaculatory process, m3. third medial cell, pm. parameres, pm ap. parameral apodeme, pr. proctiger, pt. phallic plate, M1, M2, M3. medial veins, m-cu. cross vein between medial and cubital veins, pd, pedicel, R1, R2, R3+4, R5,Rs. radial veins, r-m. cross vein between redial and medial veins, Sc. subcostal vein, sc. Scape, st. stylus. 


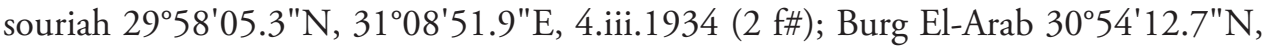

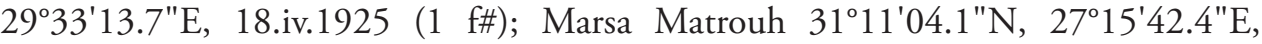
17.iii.1933 (2 f\#); Suize 29०58'09.6"N, 32³2'59.8"E, 5.iv.1927 (1 f\#); Sinai N.E. $31^{\circ} 15^{\prime} 49.4^{\prime \prime N}, 34^{\circ} 10^{\prime} 15.8^{\prime \prime E}, 19 . i v .1928$ (1 f\#); W. Silly Helwan 29०50'37.6"N, $31^{\circ} 19^{\prime} 05.0^{\prime \prime E}, 22 . i i i .1926$ (1 f\#) (MAC); Abu Rawash 3004'30.7"N, 31 ${ }^{\circ} 11^{\prime} 59.7^{\prime \prime} \mathrm{E}$, 26.iii.1952 (1 f\#); Gabal Asfar $30^{\circ} 12^{\prime} 05.7^{\prime \prime N}, 31^{\circ} 21^{\prime} 19.7^{\prime \prime E}$, 9.iii.1951 (1 f\#); Mansoura $31^{\circ} 02^{\prime} 43.1^{\prime \prime} \mathrm{N}, 31^{\circ} 22^{\prime} 54.9^{\prime \prime} \mathrm{E}, 2 . i i i .1955$ (1 f\#); Mansouriah $29^{\circ} 58^{\prime} 05.3^{\prime \prime} \mathrm{N}$,

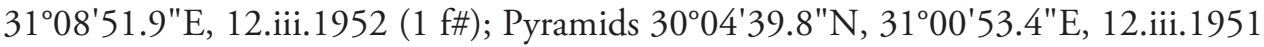
(1 f\#); W. Natroun 30²5'58.2"N, 30¹4'39.2"E, 2.iv.1951 (1 f\#) (ASUC).

Specimens previously identified as $N$. fascifrons: Abu Rowash $30^{\circ} 04^{\prime} 30.7^{\prime \prime N}$,

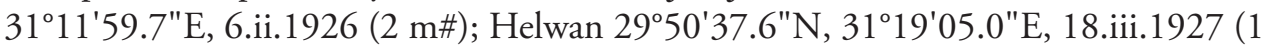

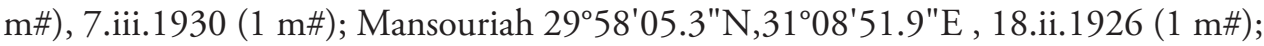

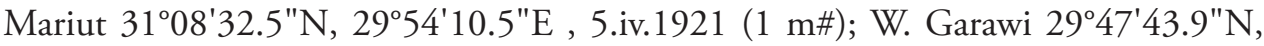

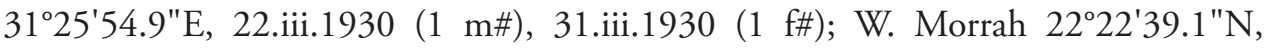
$33^{\circ} 46^{\prime} 00.3^{\prime \prime E}$, 26.iii.1927 (1 m\#) (AZUC); Kafr Hakim 3004'39.7"N, 31 066'46.3"E, 20.iii.1926 (1 m\#); Mansouriah 29 $58^{\prime} 05.3^{\prime \prime} \mathrm{N}, 31^{\circ} 08^{\prime} 51.9^{\prime \prime E}, 13 . i i .1926$ (1 m\#), 2.iii.1927 (1 m\#); W. Garawi 29 $47^{\prime} 43.9^{\prime \prime N}, 31^{\circ} 25^{\prime} 54.9^{\prime \prime E}$, 22.iii.1930 (1 m\#), 31.iii.1930 (2 m\#) (CUC); Dekheila 3107'32.0"N, 2948'37.3"E, 4.ii.1928 (2 m\#), 4.iii.1928 (1 m\#); Burg El-Arab 3054'12.7"N, 29³3'13.7"E, 19.iv.1923 (1 m\#); Kerdasa $30^{\circ} 01^{\prime} 32.1^{\prime \prime N}, 31^{\circ} 06 ' 27.5 " E, ~ 15 . i i .1923$ (1 m\#), 10.ii.1925 (1 m\#); Mansouriah 2958'05.3"N, 310' 51.9"E, 13.ii.1926 (1 m\#), 6.iii.1926 (1 m\#); Burg El-Arab 30 54'12.7"N, 29³3'13.7"E, 27.iv.1923 (1 m\#), 18.iv.1925 (5 m\#); (Six Towers) Suize Road 29'59'45.9"N, 32²9'34.4"E , 26.iii.1926 (1 m\#); Gabal Asfar 30¹2'05.7"N,

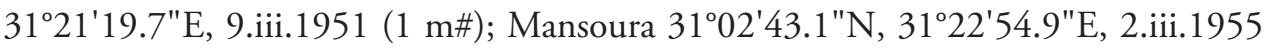
(1 m\#) (ASUC).

Specimens previously identified as $N$. abdominalis: Egypt (1 f\#), without date, specimen number USNMENT01371553 (USNM) (previously identified by W. Wirth as $N$. abdominalis but as $N$. ater by Dr. Torsten Dikow using our key).

Diagnosis. Frons shiny black with a transverse white band; wing with small cells extending forward from R1 to hind margin; abdomen orange with longitudinal black vitta in male but entirely black in female.

Redescription. Length: male body $10-16 \mathrm{~mm}$, wing $9-15 \mathrm{~mm}$. Female body $14-$ $21 \mathrm{~mm}$, wing 10-13 mm. Male: Frons shiny black with transverse white band; face rather short, snout-like, sides with grayish yellow pollinosity (Figs 23-25). Antenna blackish and pollinose (Fig. 26).

Thorax black or blackish brown, with blackish to yellowish brown hairs, pleurae with long and dense black hairs; leg blackish or dark yellow; claws well developed; pulvilli almost rudimentary. Wing blackish brown, but apex and postero-apical margin pale brown; wing with many small cells extend forward from R1 to hind margin (Fig. 27). Abdomen short, wide, reddish to orange with longitudinal black strip that is narrow posteriorly and sometimes absent at apex (Fig. 28). Male genitalia with gonocoxite having two processes, inner process short and slender, whereas the outer process is longer, thicker and subapically curved; gonostyli longer than the inner gonocoxal processes but shorter than the outer one, with subapical cleft and small projection (Figs 


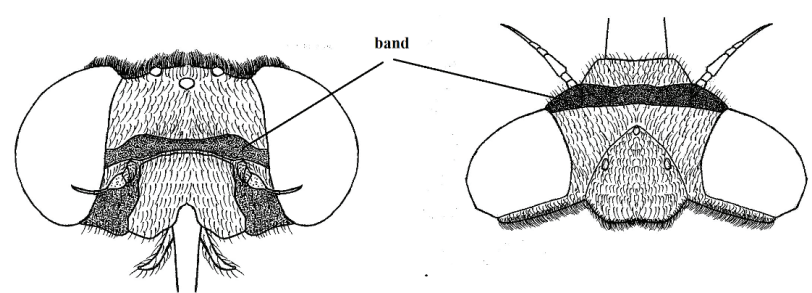

33
34

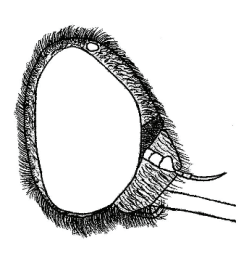

35

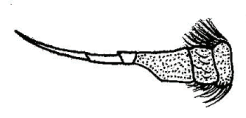

36

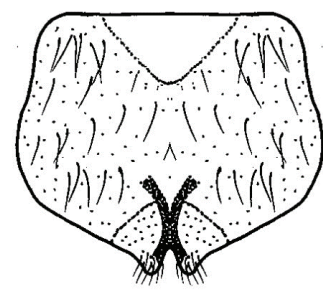

39

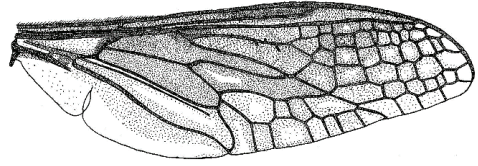

37

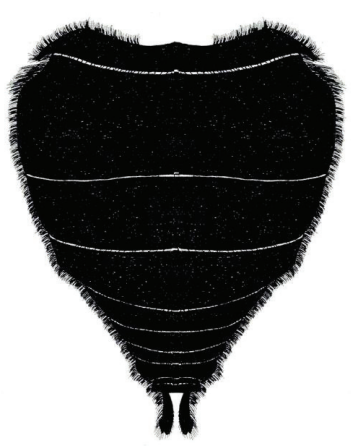

38

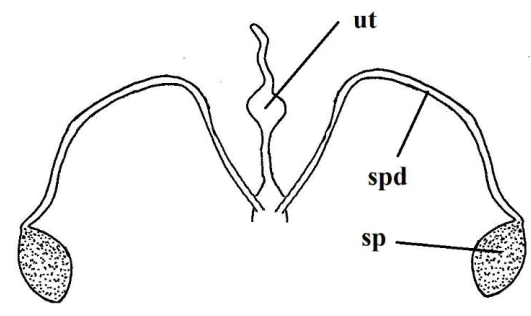

40

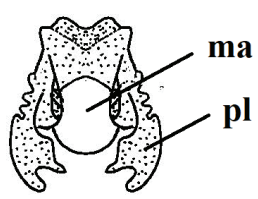

41

Figures 33-4I. Female of Nemestrinus ater, head, frontal (33), head, dorsal (34), head, lateral (35), antenna (36), wing (37), and abdomen (38). 39-4I female genitalia: subgenital plate (39), genital furca (40), and spermathecae (4I). Abbreviations: ma. median aperture, pl. posterolateral projection, sp. spermatheca, spd. spermathecal duct, ut. uterus.

29, 30); aedeagus free, narrow distally and fused proximally with parameres; parameres slightly sinuate; parameral apodeme a long, while aedeagal tine is short; ejaculatory apodeme slender and narrow (Figs 31, 32). Female. Similar as male (Figs 33-37), except: eyes widely separated more than in male. Abdomen entirely black or at least with reddish black lateral margins (Fig. 38). Head in male slightly wider than thorax but in female narrower than thorax. Female genitalia with quadrate subgenital plate, bilobed distally (Fig. 39); genital furca with furcated arms and serrated laterally (Fig. 40); uterus small, with terminal accessory process; spermathecal ducts narrow and long with oval medium spermathecae (Fig. 41). 


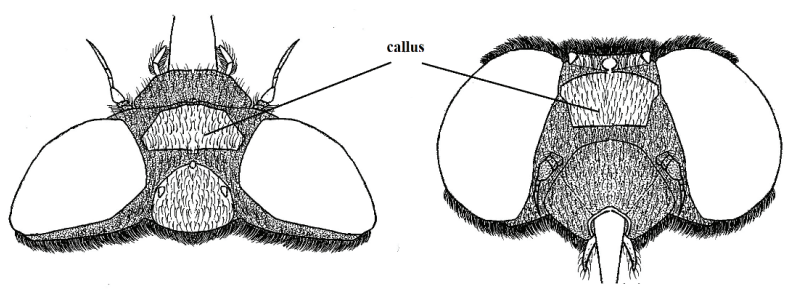

42
43

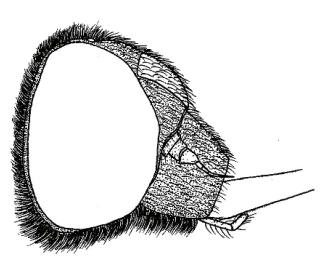

44

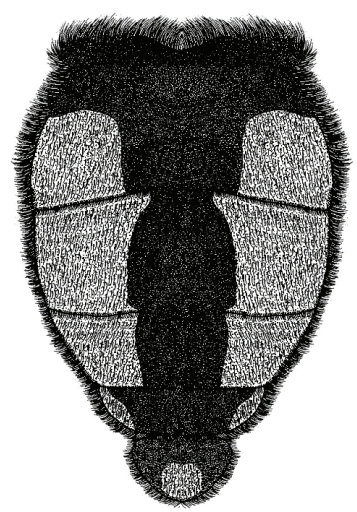

47

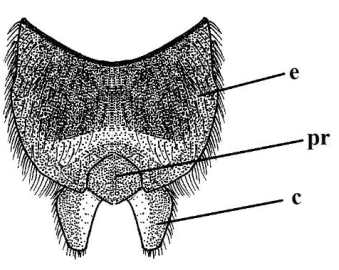

48

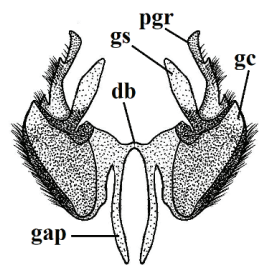

49

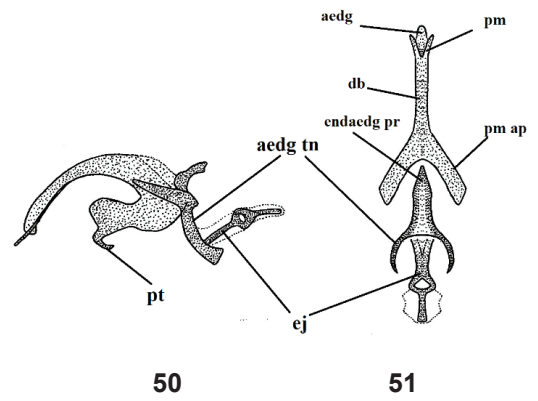

50

Figures 42-5 I. Male of Nemestrinus exalbidus, head, frontal (42), head, dorsal (43), head, lateral (44), antenna (45), wing (46), and abdomen (47). 48-5 I male genitalia: epandrium, proctiger, and cerci (48), gonocoxite with gonostylus, ventral (49), aedeagal complex, lateral (50) and dorsal (5I). Abbreviations: aedg. aedeagus, aec. aedeagal complex, aedg tn. aedeagal tine A2. anal vein, al. alula, bm. basal medial cell, br. basal radial cell, c. cerci, C. costa, CuA1,2, CuP. cubital veins, d. discal cell, db. dorsal bridge, DV. diagonal vein, e. epandrium, ej. ejaculatory apodeme, endaedg pr. endoaedeagal process, 1 st $\mathrm{fl}$. first flagellomere, gap. gonocoxal apodeme, gc. gonocoxite, gpr. gonocoxal process, gs. gonostylus, h. hypandrium, h. humeral cross vein, lat ej pr. lateral ejaculatory process, m3. third medial cell, pm. parameres, pm ap. parameral apodeme, pr. proctiger, pt. phallic plate, M1, M2, M3. medial veins, $\mathrm{m}$-cu. cross vein between medial and cubital veins, pd, pedicel, R1, R2, R3+4, R5, Rs. radial veins, r-m. cross vein between redial and medial veins, Sc. subcostal vein, sc. Scape, st. stylus. 
Local distribution. Coastal strip, Lower Nile.

Geographical distribution. Algeria, Egypt, Ethiopia, Israel, Spain, and Tunisia (Sack 1933; Bernardi, 1973; Richter 1988).

Remarks. Nemestrinus abdominalis and $N$. fascifrons are newly synonymized with $N$. ater. The earlier works of Lichtwardt (1909, 1919), Villeneuve (1912), and Bequaert (1938) suggested that $N$. ater, $N$. abdominalis, and $N$. fascifrons were closely related to each other based on Egyptian, Tunisian, and Palestinian material. We observed that $N$. ater has sexually dimorphic abdominal color. We also discovered that all the specimens previously identified by Efflatoun as $N$. fascifrons are males and we confirm these identifications. We also confirm that all the specimens that were previously identified by the same author as $N$. ater are females and confirmed by us as $N$. fascifrons. The two "species" of Efflatoun were captured from approximately the same locality and time of year by the same collector, i.e., "Efflatoun collected males at W. Garawi on 22.iii.1930 and 31.iii.1930 and females at W. Garawi on 22.iii.1930 and 31.iii.1930"; both are deposited in the Cairo University collection. We observed the sexual dimorphism and regard them as representing the same species.

\section{Nemestrinus exalbidus (Lichtwardt, 1907)}

Figures 42-60

Nemestrina exalbidus Lichtwardt, 1907: 441. Type locality: Israel (Jerusalem).

Specimens examined. W. Dar El-Maskhara $29^{\circ} 47^{\prime} 02.9^{\prime \prime} \mathrm{N}, 31^{\circ} 24^{\prime} 59.9^{\prime \prime} \mathrm{E}, 12 . i v .1930$

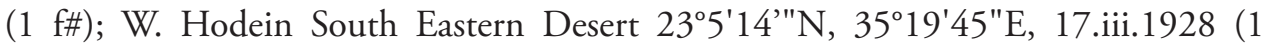
m\#); W. Hoff 2953'02.6"N, 31¹8'42.2"E, 12.iv.1921 (1 f\#), 24.iii.1930 (1 m\#); W. Zohleiga $26^{\circ} 07^{\prime} 59.9^{\prime \prime} \mathrm{N}, 33^{\circ} 45^{\prime} 00.0^{\prime \prime E}$, $27 . i i i .1925$ (1 f\#) (AZUC); Abu Rowash $30^{\circ} 04^{\prime} 30.7^{\prime \prime N}, 31^{\circ} 11^{\prime} 59.7^{\prime \prime E}$, 16.iii.1927 (1 f\#); Ogret El-Sheikh 2952'50.1"N,

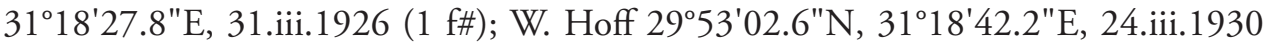

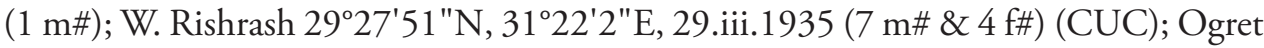

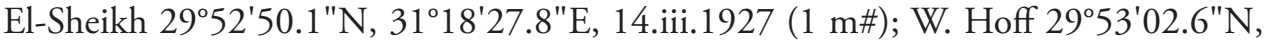
$31^{\circ} 18^{\prime} 42.2^{\prime \prime E}, 30 . i i i .1928$ (1 m\#); W. Zohleiga 260' $59.9^{\prime \prime N}, 33^{\circ} 45^{\prime} 00.0 " \mathrm{E}, 25,29$. iii.1925 (1 f\#) (MAC); Etaka 2926'19.1"N, 32²8'07.2"E , 22.ii.1951(1 f\#), 26.iii.1951 (1 m\#); Kerdasa $30^{\circ} 01^{\prime} 32.1 " \mathrm{~N}, 31^{\circ} 06^{\prime} 27.5^{\prime \prime E}, 14 . i v .1951$ (3 f\#); Mansouriah $29^{\circ} 58^{\prime} 05.3^{\prime \prime} \mathrm{N}, 31^{\circ} 08^{\prime} 51.9^{\prime \prime} \mathrm{E}, 25 . \mathrm{iv} .1957$ (1 m\#); W. Kaber 23²6'29"N, 2550'23"E, 1.iv.1994 (1 f\#) (ASUC).

Diagnosis. Frons covered with dense gray pollinosity except shiny black oval callus below ocellar triangle; wing hyaline, except slightly brownish along anterior margin, with a few small cells extending forward from R2 +3 to M1 or M2. Abdomen orange or reddish with longitudinal median black vitta.

Redescription. Length: male body $14-17 \mathrm{~mm}$, wing $12.5-14.5 \mathrm{~mm}$. Female body $18 \mathrm{~mm}$, wing $15.5 \mathrm{~mm}$. Head shiny black with white hairs; frons covered with dense gray pollinosity except shiny black oval callus below ocellar triangle; face rather conical 


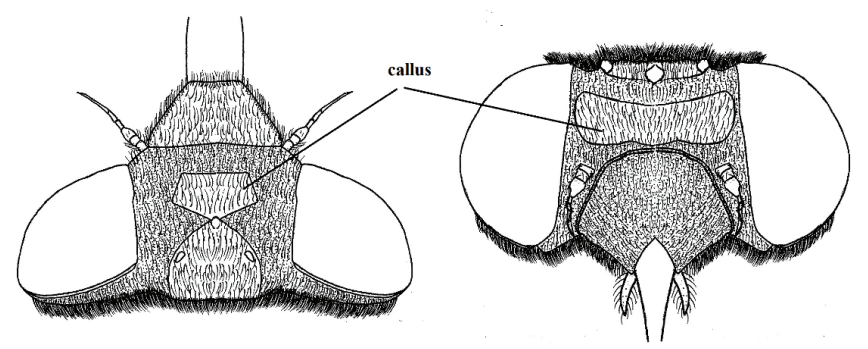

52
53

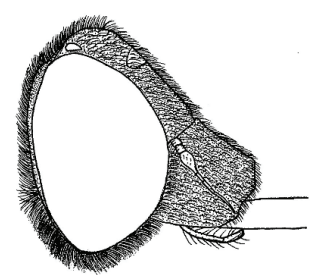

54

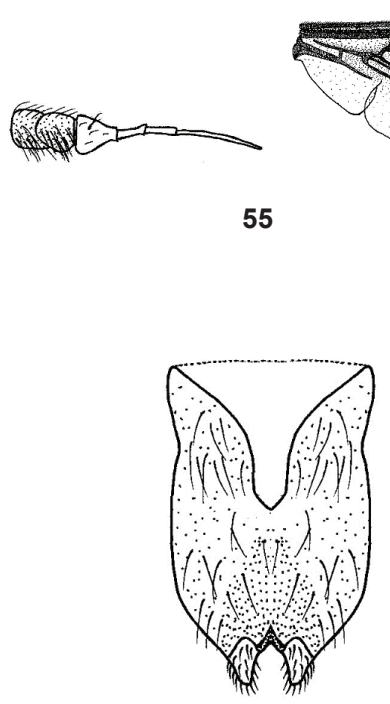

58

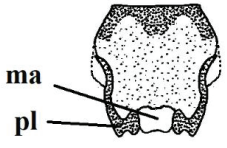

59

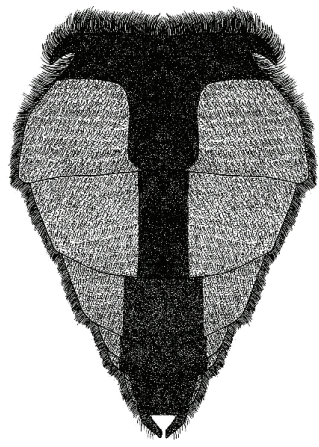

57

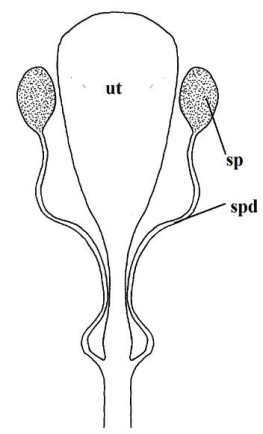

60

Figures 52-60. Female of Nemestrinus exalbidus, head, frontal (52), head, dorsal (53), head, lateral (54), antenna (55), wing (56), and abdomen (57). 58-60 female genitalia: subgenital plate (58), genital furca (59), and spermathecae (60). Abbreviations: ma. median aperture, pl. posterolateral projection, sp. spermatheca, spd. spermathecal duct, ut. uterus.

(Figs 42-44, 52-54). Thorax shiny black with dense whitish hairs laterally and a few dorsally. Leg orange, but femora black. Wing hyaline, except pale brownish anterior margin, with just a few small cells extending forward from R2+3 to M1 or M2 (Figs $46,56)$. The differences in cell number and structure on the wing is continuous variation and inconsistently different between males and females. Abdomen orange or reddish with longitudinal black median vitta; base of abdomen covered with dense, short, 
yellowish gray hairs, lateral margins of subsequent segments with dense white hairs; venter of abdomen entirely orange (Figs 47,57 ). Gonocoxite with only inner gonocoxal process, tapered apically (Figs 48, 49); distiphallus narrow; parameral apodeme rather short, aedeagal tine narrow and curved, forming semicircle, pointed distally; ejaculatory apodeme distally broader (Figs 50, 51). Female differentiated from male by the eyes that are more dichoptic. Female genitalia with rectangular subgenital plate, excavated proximally to approx. $1 / 2$ length of plate (Fig. 58); genital furca with small genital aperture, between projections with broad ends, and curved posteromedially with small curve on upper and lower margins (Fig. 59); uterus large and flatted, spermathecae long (Fig. 60).

Local distribution. Eastern Desert, Lower Nile.

Geographical distribution. Egypt, Iran, and Israel (Sack 1933; Bernardi, 1973; Richter 1988).

\section{Nemestrinus fasciatus (Olivier, 1811)}

Figures 61-79

Nemestrina fasciata Olivier, 1811: 171-172. Type locality: Egypt.

Specimens examined. Burg El-Arab 3054'12.7"N, 29³3'13.7"E, 6.v.1926 (1 m\#); Burg El-Arab, 2.v.1921 (10 m\#) (AZUC); Burg El-Arab 3054'12.7"N, 29³3'13.7"E, 5.v.1926 (1 f\#) (CUC); Burg El-Arab 3054'12.7"N, 29³3'13.7"E, 10.v.1927 (1 m\#), 19.iv.1928 (6 m\# \& 6 f\#); King Mariut 3057'27.2"N, 29³8'51.0"E, 14.iv.1915 (1 m\#), 23.v.1925 (1 m\#); Burg El-Arab 3054'12.7"N, 29³3'13.7"E, 2.v.1924 (1 f\#) (MAC); Max 3109' 50.5"N, 2951'47.7"E, 21.iv.1952 (1 f\#) (ASUC).

Diagnosis. Frons and face with dense whitish hairs and pollinose; inner ends of transverse suture with two white spots; wing hyaline with brownish base, veins yellowish, without additional small cells; abdomen black with transverse white bands, slightly curved medially; gonocoxite with inner and outer processes, the inner tapered apically, outer slightly curved subapically; gonostyli broader than gonocoxal processes with broad subapical projection; aedeagal complex narrow, aedeagus slightly broader distally.

Redescription. Length: male body $13-16.5 \mathrm{~mm}$, wing $1-12 \mathrm{~mm}$. Female) body $12-19.5 \mathrm{~mm}$, wing $1-13 \mathrm{~mm}$.

Head triangular in profile, ventrally with dense, short, whitish hairs; frons and face with dense whitish hairs and pollinosity (Figs 61-63, 71-73); antenna distinctly jointed, stylus is brown (Figs 64, 74). Thorax slightly shiny black; inner parts of transverse suture with two white spots; scutellum and mesonotum with grayish yellow hairs; pleurae with long white hairs. Leg with blackish femora covered with whitish hairs; tibiae and tarsi brown with brownish red hairs; pulvilli orange, nearly as long as claws. Wing hyaline with brownish infuscate base; veins yellowish, without additional small cells (Figs 65, 75). Abdomen black with transverse white bands, whi slightly curved medially; basal segments with long yellowish hairs but subsequent segments with white hairs; venter of abdomen with dense white hairs that fold on the lateral 


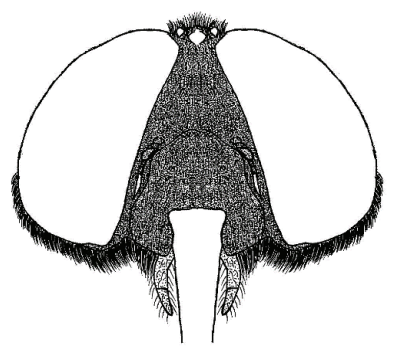

61

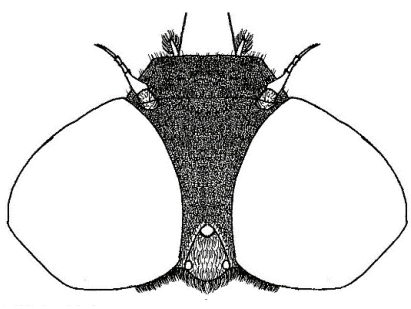

62

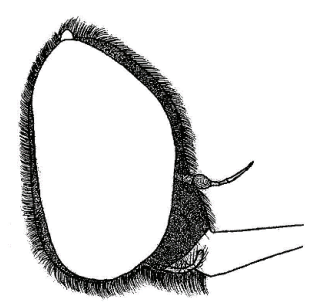

63

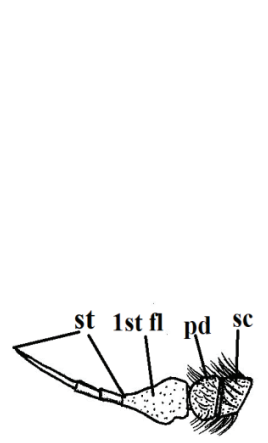

64

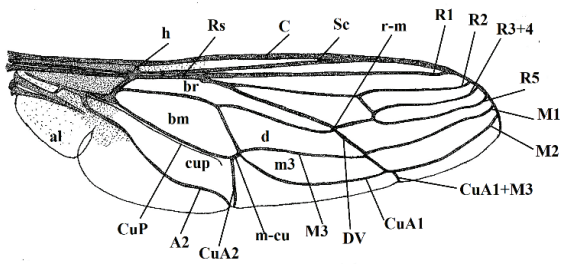

65

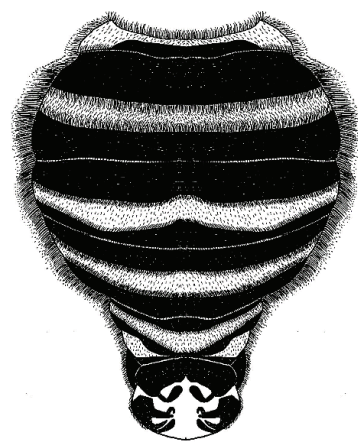

66

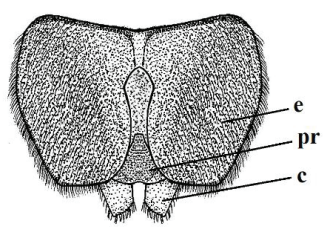

67

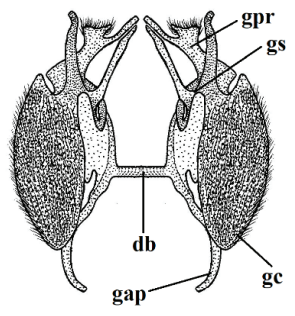

68

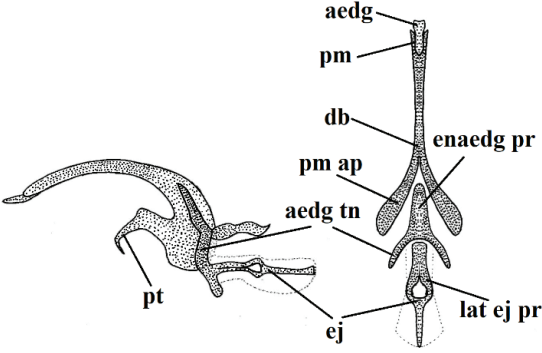

69

Figures 6 I-70. Male of Nemestrinus fasciatus, head, frontal (6I), head, dorsal (62), head, lateral (63), antenna (64), wing (65), and abdomen (66). 67-70 male genitalia, epandrium, proctiger and cerci (67), gonocoxite with gonostylus, ventral (68), aedeagal complex, lateral (69) and dorsal (70). Abbreviations: aedg. aedeagus, aec. aedeagal complex, aedg tn. aedeagal tine A2. anal vein, al. alula, bm. basal medial cell, br. basal radial cell, c. cerci, C. costa, CuA1,2, CuP. cubital veins, d. discal cell, db. dorsal bridge, DV. diagonal vein, e. epandrium, ej. ejaculatory apodeme, enaedg pr. endoaedeagal process, 1 st fl. first flagellomere, gap. gonocoxal apodeme, gc. gonocoxite, gpr. gonocoxal process, gs. gonostylus, h. hypandrium, h. humeral cross vein, lat ej pr. lateral ejaculatory process, m3. third medial cell, pm. parameres, pm ap. parameral apodeme, pr. proctiger, pt. phallic plate, M1, M2, M3. medial veins, m-cu. cross vein between medial and cubital veins, pd, pedicel, R1, R2, R3+4, R5, Rs. radial veins, r-m. cross vein between redial and medial veins, Sc. subcostal vein, sc. Scape, st. stylus. 


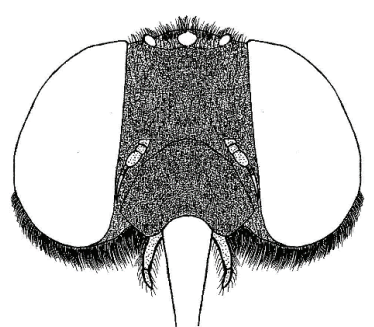

71

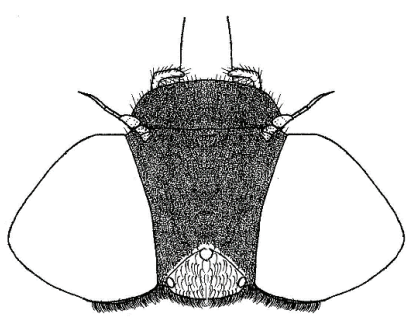

72

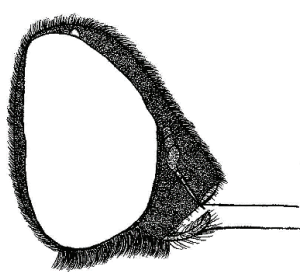

73

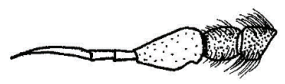

74

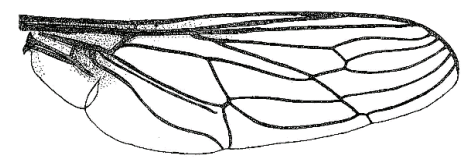

75

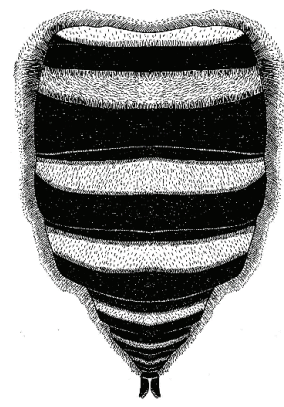

76

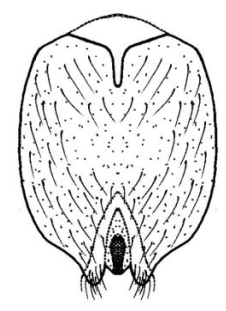

77

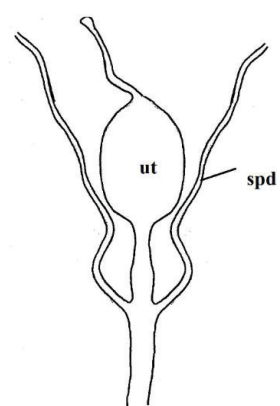

78

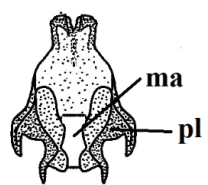

79

Figures 7I-79. Female of Nemestrinus fasiciatus, head, frontal (7I), head, dorsal (72), head, lateral (73), antenna (74), wing (75), and abdomen (76). 77-79 female genitalia: subgenital plate (77), genital furca (78), and spermathecae (79). Abbreviations: ma. median aperture, pl. posterolateral projection, sp. spermatheca, spd. spermathecal duct, ut. uterus.

margins (Figs 66, 76). Gonocoxite with inner and outer processes, inner tapered apically, outer slightly curved subapically; gonostyli broader than gonocoxal processes with broad projection subapically (Figs 67, 68); aedeagal complex narrow, aedeagus slightly broader apically (Figs 69, 70).

Female: eyes separated in both sexes but considerably broader than in male at vertex; genitalia with sub-rectangular subgenital plate (Fig. 77); genital furca narrower 
anteriorly with four incurved posterolateral projections (Fig. 78); uterus with terminal accessory process; spermathecae rather long (Fig. 79).

Local distribution. Coastal strip.

Geographical distribution. Algeria, Egypt, Morocco, Israel, and Syria (Sack 1933; Bernardi, 1973; Richter 1988).

\section{Nemestrinus reticulatus Latreille, 1802}

Figures $80-85$

Nemestrinus reticulatus Latreille, 1802: 437. Type locality: not given but according to Latreille (1809: 307), it is Egypt and Syria.

Rhynchocephalus latreillei Fischer, 1812: 195.

Nemestrina cinctus Macquart, 1840: 16.

Nemestrina kindermanni Bischof, 1905: 172.

Diagnosis. Frons with shiny yellow or black spot below ocelli; mesonotum with two gray spots at inner ends of the transverse suture, between them there is a thin longitudinal stripe; wing hyaline in posterior $1 / 2$ and apex but brownish on anterior $1 / 2$ and slightly infuscate at base; wing with small cells that extend forward from $R_{2}$ to hind margin; abdomen gray, matte, with incomplete transverse black stripes; tergite II bears shiny black spots divided in the middle by a transverse longitudinal gray strip; black spots on tergites III-V more or less fused into bands with an emargination along the posterior margin; on tergite III, gray emargination varies from very deep to nearly absent; abdominal venter with dense gray pollinosity, the second sternite with central black spot.

Description. Length: body $14-15 \mathrm{~mm}$. Head black with dense gray pollinosity and whitish hairs; frons with shiny yellow or black spot below ocelli, in male frons at vertex nearly as wide as eye width (Figs 80, 83), while in female nearly twice as eye width (Fig. 81); antenna with orange scape and pedicel, first flagellomere brown to blackish brown with some gray pollinosity (Fig. 82), basal two segments of stylus subequal in length and segment III $2 / 3 \times$ longitudinal eye diameter; palpi yellow or brown with black apices. Thorax pale black with yellowish white or grayish white hairs, but longer and denser on scutellum and pleurae; mesonotum with two gray spots at inner ends of transverse suture and between them is a thin longitudinal stripe. Leg rusty red; in females, only hind tarsi blackish or hind leg entirely blackish; in males, all femora black; hind tibiae and tarsi blackish. Wing hyaline over posterior $1 / 2$ and at apex, but somewhat brown over anterior $1 / 2$ and slightly infuscate at base; wing with small cells that extend forward from $\mathrm{R}_{2}$ to hind margin (Fig. 85). Abdomen gray, matte, with incomplete transverse black stripes; tergite II with shiny black spots divided in the middle by a transverse longitudinal gray strip; black spots on tergites III-V are more or less fused into bands with an emargination along the posterior margin; on tergite III, gray emargination varies from very deep to nearly absent; abdominal venter with dense gray pollinosity, sternite II with black central spot (Fig. 84). 


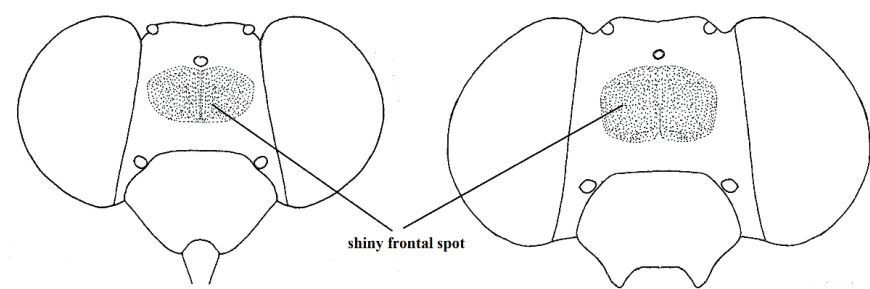

80

81

82

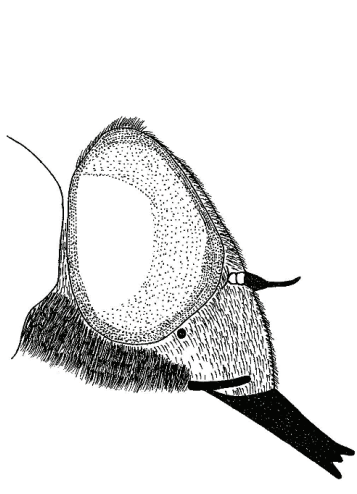

83

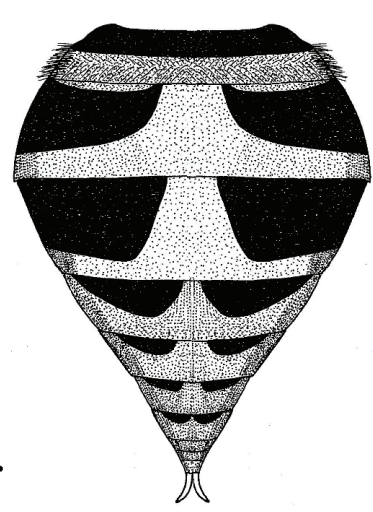

84

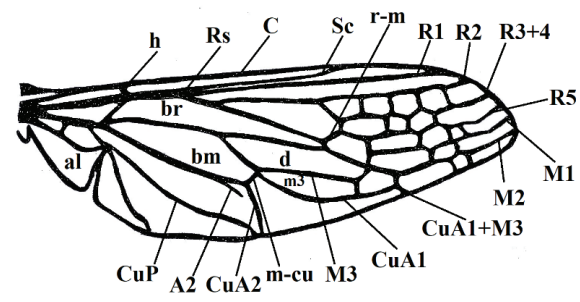

85

Figures 80-85. Nemestrinus reticulatus, male head, frontal (80), female head, frontal (81), male antenna (after Bequaert, 1938) (82), male head, lateral (83), female abdomen (after Sack 1933) (84), and wing (after Seguy, 1926) (85). Abbreviations: A2. anal vein, al. alula, bm. basal medial cell, br. basal radial cell, C. costa, CuA1,2, CuP. cubital veins, d. discal cell, DV. diagonal vein, h. humeral cross vein, m3. third medial cell, M1, M2, M3. medial veins, m-cu. cross vein between medial and cubital veins, R1, R2, R3+4, R5,Rs. radial veins, r-m. cross vein between redial and medial veins, Sc. subcostal vein.

Local distribution. Unknown.

Geographical distribution. Armenia, Egypt, Greece, Russia (Caucasus), Saudi Arabia, Syria, and Turkey (Sack 1933; Bernardi, 1973).

Remarks. This species is not represented in Egyptian collections nor in the field. We include the species here and in the key below as it has been recorded from Egypt (Sack 1933 \& Bernardi, 1973 \& Richter 1988); future research might reveal its presence in this part of Africa. 


\section{Nemestrinus rufipes (Olivier, 1811)}

Figures 1, 2, 86-104

Nemestrina rufipes Olivier, 1811: 171.

Nemestrina lateralis Wiedemann, 1828: 560.

Nemestrina ruficornis Macquart, 1840: 15. Syn. nov.

Type material. Nemestrinus rufipes: Type male, "Aegypten", Egypt (ZMHB) (pers. comm. Mr. Sven Marotzke). Nemestrina lateralis: Type female, "Aegypten", Egypt (ZMHB) (pers. comm. Mr. Sven Marotzke).

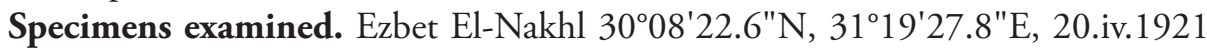

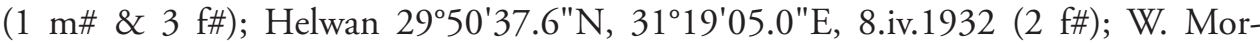

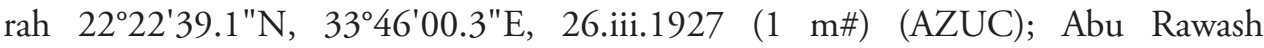
3004'30.7"N, 31 $11^{\circ} 59.7^{\prime \prime E}$ 13.iii.1955 (3 m\# \& 4 f\#), 17.iii.1955 (1 m\#); Kerdasa

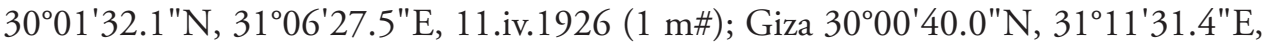

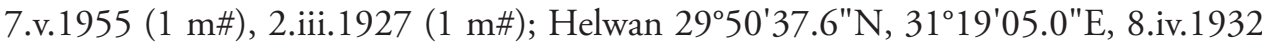
(2 f\#), 3.iv.1934 (1 m\# \& $3 \mathrm{f}$ ), 8.iv.1934 (6 f\#), 17.iii.1934 (1 f\#), 17.iv.1934 (1 f\#), 23.iv.1935 (3 f\#); W. Garawi 2947'43.9"N, 31 25'54.9"E, 31.iii.1930 (1 f\#) (CUC); Abu Rawash 3004'30.7"N, 31¹1'59.7"E, 11.iv.1925 (1 m\#), 17.iv.1925 (2 m\# \&

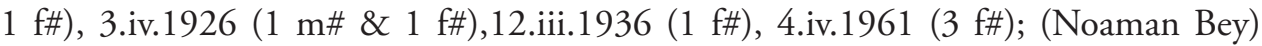
Alexandria $31^{\circ} 10^{\prime} 03.5^{\prime \prime} \mathrm{N}, 29^{\circ} 51^{\prime} 56.2^{\prime \prime E}$, (1 f\#, without date); Assyut $27^{\circ} 23^{\prime} 00.0^{\prime \prime} \mathrm{N}$,

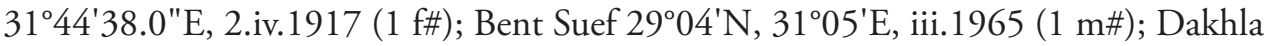

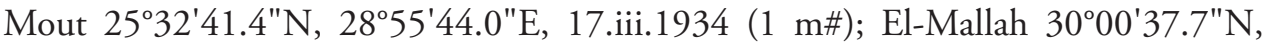

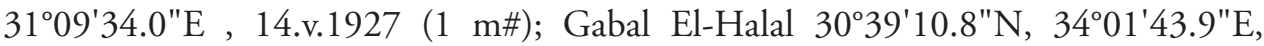
25.iv.1924 (1 m\# \& $1 \mathrm{f \# );} \mathrm{Gabal} \mathrm{El-Sanadiq,} \mathrm{5.iv.1934} \mathrm{(1} \mathrm{f \# );} 12 . i v .1924$ (1 m\#); Kafr Hakim 3004'39.7"N, 31 06'46.3"E, 7.iv.1924 (1 m\#), 14.iv.1925 (1 m\#), 20.iv.1925

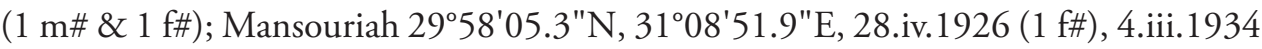

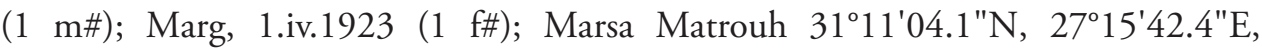
19.i.1933 (1 m\#), 17.iii.1933 (1 m\#), 1.iv.1961 (1 f\#); W. Garawi 29०47'43.9"N,

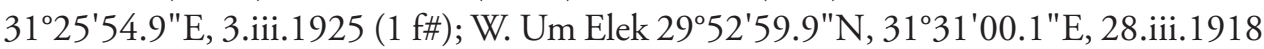
(1 f\#); W. Zohleiga 2607'59.9"N, 3345'00.0"E, 25-29.iii.1925 (1 f\#) (MAC); W. Digla $29^{\circ} 59^{\prime} 00.1 " \mathrm{~N}, 31^{\circ} 19^{\prime} 41.2^{\prime \prime E}$, 5.iv.1952 (1 m\#) (ASUC). Aegypten (1 m\# \& 1f\#), without date (ZMHB) sent by Mr. Sven Marotzke and Bernhard Schurian; Dakahlia, Mansuriya $31.1656^{\circ} \mathrm{N}, 31.4913^{\circ} \mathrm{E} 31 . i i i .1964$ (1 m\# \& $1 \mathrm{f \# )} \mathrm{specimen}$ numbers: male: USNMENT01371563, female: USNMENT01371564, Cairo, Marg $30.1543^{\circ} \mathrm{N}, 31.3484^{\circ} \mathrm{E}(1 \mathrm{f} \#)$, without date, specimen number USNMENT01371561 (USNM) (identified by Dr. Torsten Dikow).

Diagnosis. Frons covered with dense orange yellow pollinosity except with shiny blackish brown transverse oval callus below dark orange ocellar triangle; wing with yellowish brown band in the middle, but clear in apical part and along posterior margin; wing with small cells extending forward from R2 to M1 or M2. Abdomen orange to reddish orange with longitudinal black median vitta; first tergite entirely black, ter- 


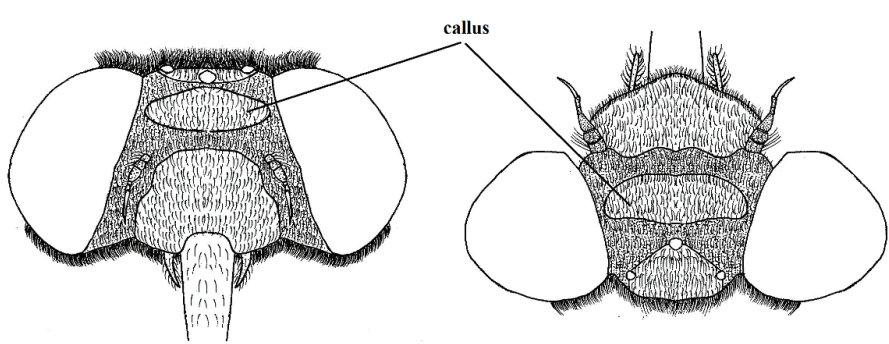

86
87

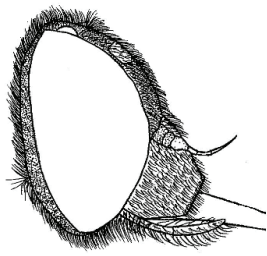

88

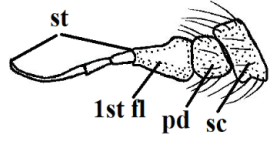

89

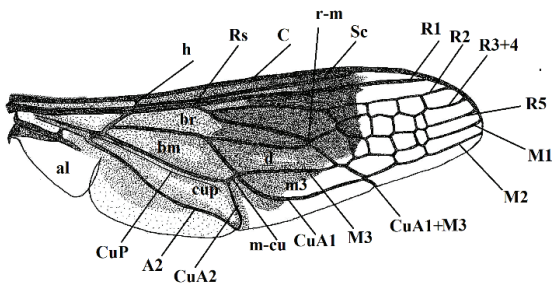

90

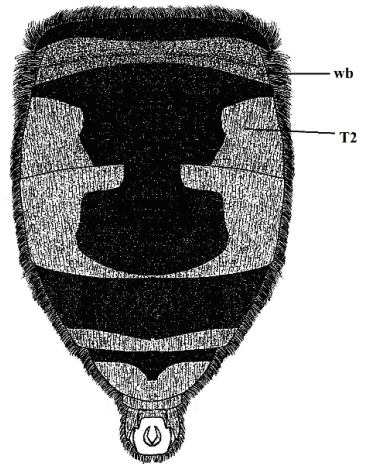

91

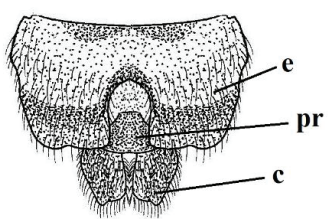

92

Figures 86-95. Male of Nemestrinus rufipes, head, frontal (86), head, dorsal (87), head, lateral (88), antenna (89), wing (90), and abdomen (91). 92-95 male genitalia: epandrium, proctiger and cerci (92), gonocoxite with gonostylus, ventral (93), aedeagal complex, lateral (94) and dorsal (95). Abbreviations: aedg. aedeagus, aec. aedeagal complex, aedg tn. aedeagal tine A2. anal vein, al. alula, bm. basal medial cell, br. basal radial cell, c. cerci, C. costa, CuA1,2, CuP. cubital veins, d. discal cell, db. dorsal bridge, DV. diagonal vein, e. epandrium, ej. ejaculatory apodeme, enaedg pr. endoaedeagal process, 1 st fl. first flagellomere, gap. gonocoxal apodeme, gc. gonocoxite, gpr. gonocoxal process, gs. gonostylus, h. hypandrium, h. humeral cross vein, lat ej pr. lateral ejaculatory process, m3. third medial cell, pm. parameres, pm ap. parameral apodeme, pr. proctiger, pt. phallic plate, M1, M2, M3. medial veins, m-cu. cross vein between medial and cubital veins, pd, pedicel, R1, R2, R3+4, R5, Rs. radial veins, $r-m$. cross vein between redial and medial veins, Sc. subcostal vein, sc. Scape, st. stylus, T2. tergite 2, wb. White band. 


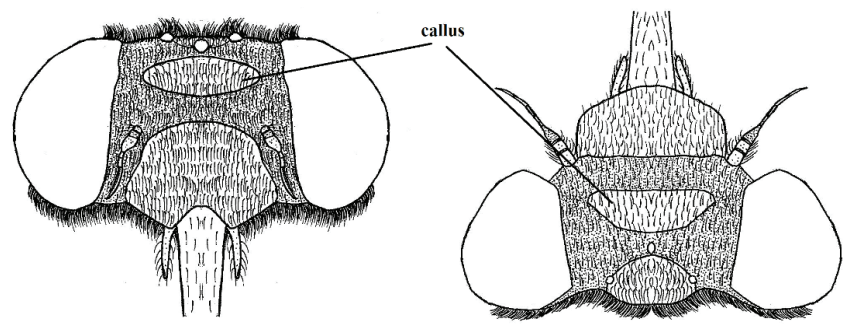

96
97

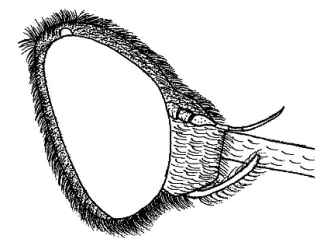

98

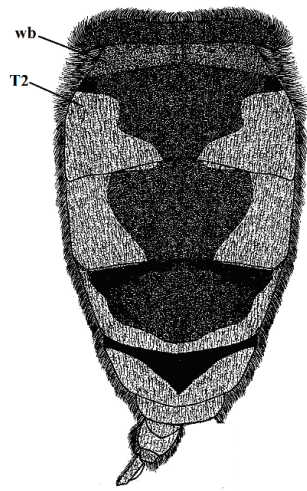

101
99

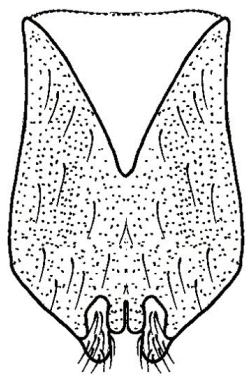

102

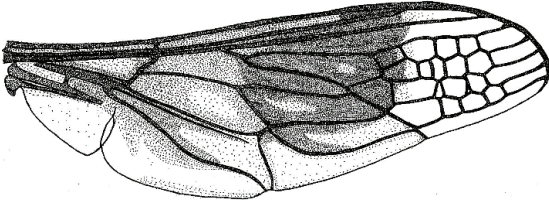

100

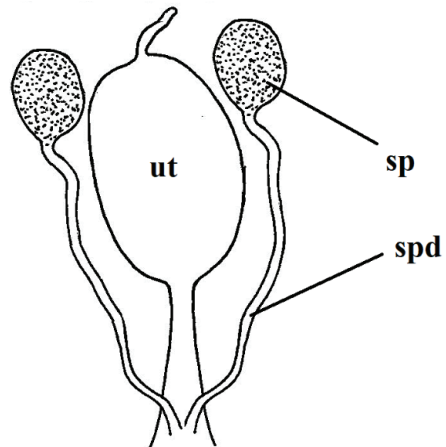

103

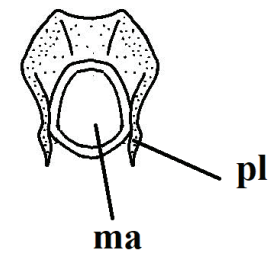

104

Figures 96-104. Female of Nemestrinus rufipes, head, frontal (96), head, dorsal (97), head, lateral (98), antenna (99), wing ( 100 ) and abdomen ( 101 ). I 02-1 04 female genitalia: subgenital plate (I 02), genital furca (103), and spermathecae (104). Abbreviations: ma. median aperture, pl. posterolateral projection, sp. spermatheca, spd. spermathecal duct, ut. uterus, T2. tergite 2, wb. White band.

gite II on anterior margin with transverse white band. Gonocoxite with inner process slightly tapered; gonostyli longer than gonocoxal process, curved subapically with small projection; aedeagus fused proximally with parameres and separated distally, parameres and aedeagus with small indentations distally in lateral view. 
Redescription. Length: male body13.5-18.5 mm, wing 11.5-16 mm. Female body 14-21 mm, wing 12-17.5 mm. Head short, wider than thorax; frons covered with dense orange-yellow pollinosity except with shiny blackish brown transverse oval callus (Figs 86-88, 96-98); face shiny brownish orange with short yellow hairs; antenna orange (Figs 89, 99); proboscis black, as long as thorax, upper surface of base with short yellow hairs; palpi orange. Thorax shiny black with yellow hairs, longer and denser on the sides and in front; mesonotum with indistinct spots at inner ends of transverse suture. Leg orange, coxae and base of femora somewhat brown, pulvilli light yellow and nearly $1 / 2$ length of claw. Wing with yellowish brown band in the middle, but clear apically and along posterior margin; wing with small cells extending forward from R2 to M1 or M2 (Figs 90, 100). Halter brown with light yellow pedicel. Abdomen orange to reddish orange with longitudinal black median vitta; tergite I entirely black, tergite II with transverse white band; abdomen with short and golden yellow hairs but longer laterally; abdominal venter orange and with black lateral margins (Figs 91, 101). Gonocoxite with inner process slightly tapered; gonostyli longer than gonocoxal process, curved subapically with small projection (Fig. 93); aedeagus fused proximally with parameres and separated distally, parameres and aedeagus with small indentations distally in lateral view (Figs 94, 95). Female genitalia: rectangular subgenital plate with large curve (Fig. 102); genital furca with large aperture surrounded by narrow and slightly curved posterolaterally projections (Fig. 103); uterus with small terminal accessory; spermathecae nearly as long as the uterus (Fig. 104).

Local distribution. Coastal strip, Lower Nile, Upper Nile, Sinai.

Geographical distribution. Algeria, Egypt, and Syria (Sack 1933; Bernardi, 1973; Richter 1988).

Remarks. Bernardi (1973) and Richter (1988) considered N. rufipes and N. ruficornis to be valid species but, in contrast, Lichtwardt (1909) and Bequaert (1938) synonymized the two. We agree with this decision based on a comparison of the original description of $N$. ruficornis (no material was examined) with the male type specimen of $N$. rufipes (Fig. 2) and the female type specimen of $N$. lateralis (Fig. 3), both in ZMHB, in addition to both sexes of many old Egyptian specimens of $N$. rufipes. Thus, we confirm that $N$. rufipes and $N$. ruficornis are conspecific and the first is the valid name, and Nemestrinus ruficornis is here synonymized with $N$. rufipes.

List of doubtful species. In the present study, three species are treated as doubtful and are excluded from the list of Egyptian Nemestrinidae: Nemestrinus caucasicus, Nemestrinus pallipes, and Nemestrinus persicus.

\section{Discussion}

As a result of this revision, we can confirm six species of Nemestrinus present in Egypt. This is lower than the 12 taxa (eleven species and one subspecies) listed by Steyskal and El-Bialy (1967) but three species are treated as doubtfully occurring including $N$. pallipes added by Bernardi (1973) and Richter (1988) as Egyptian species and three species that are newly synonymized ( $N$. abdominalis, $N$. fascifrons, and $N$. ruficornis). 


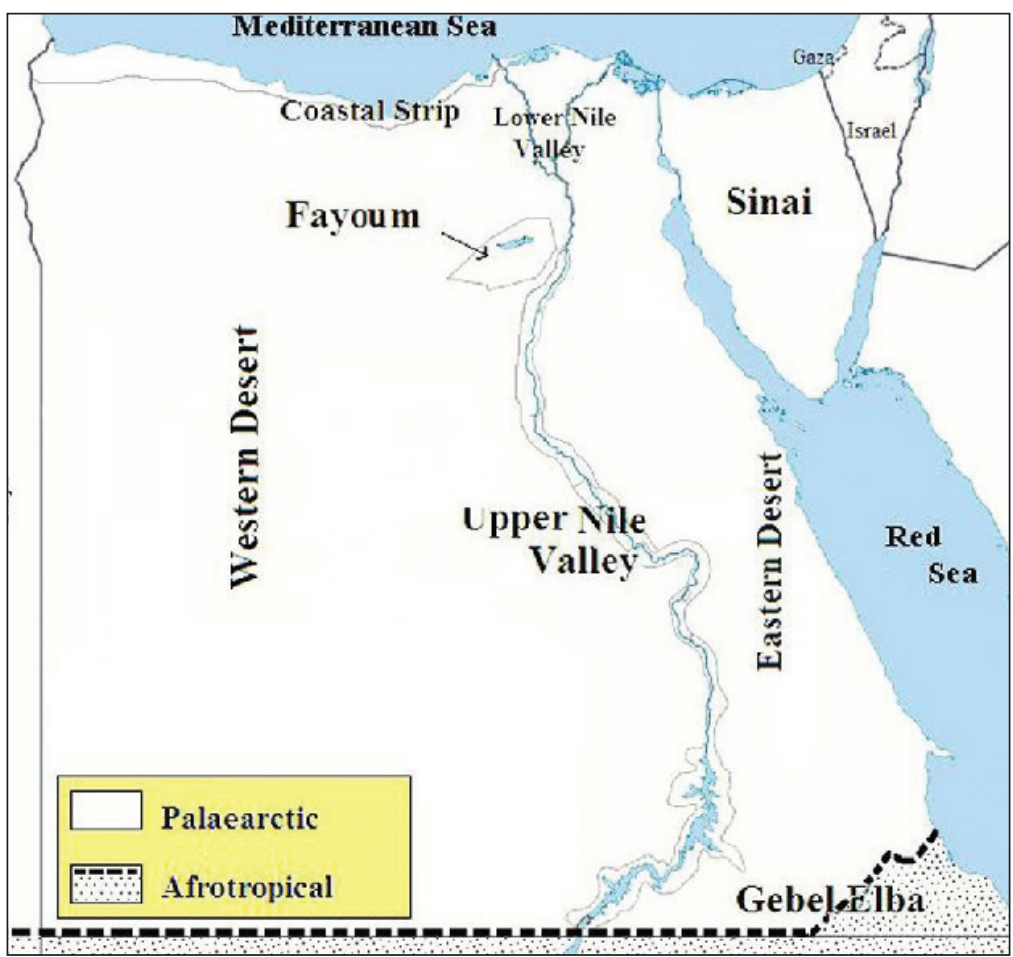

Map I. Map of Egypt showing the ecological zones (after elhawagry and gilbert 2014).

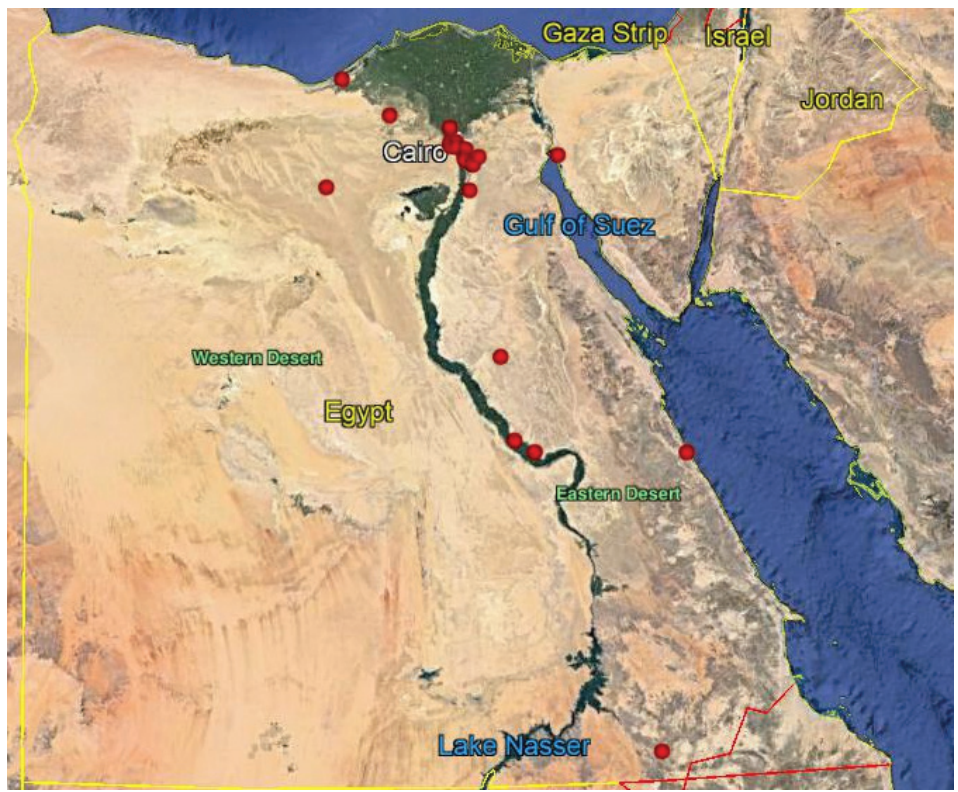

Map 2. Distribution of the species N. aegyptiacus in Egypt. 


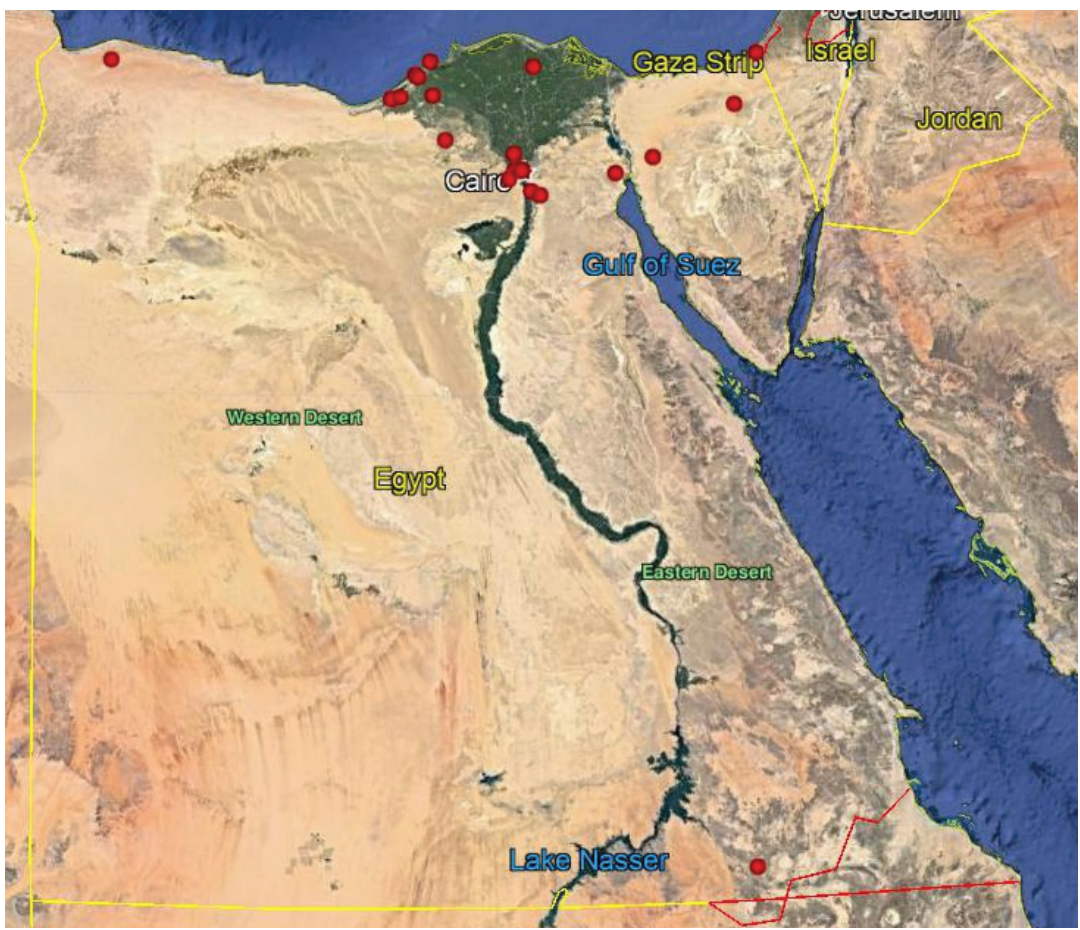

Map 3. Distribution of the species $N$. ater in Egypt.

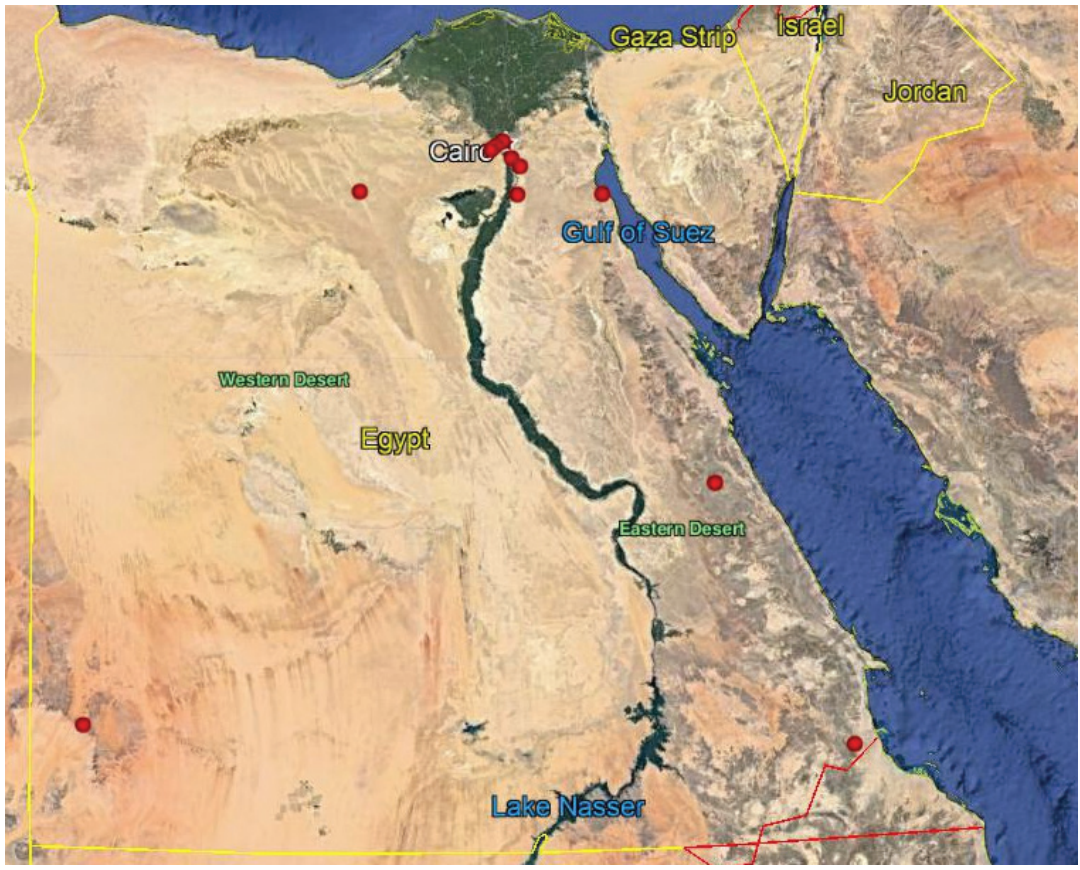

Map 4. Distribution of the species N. exalbidus in Egypt. 


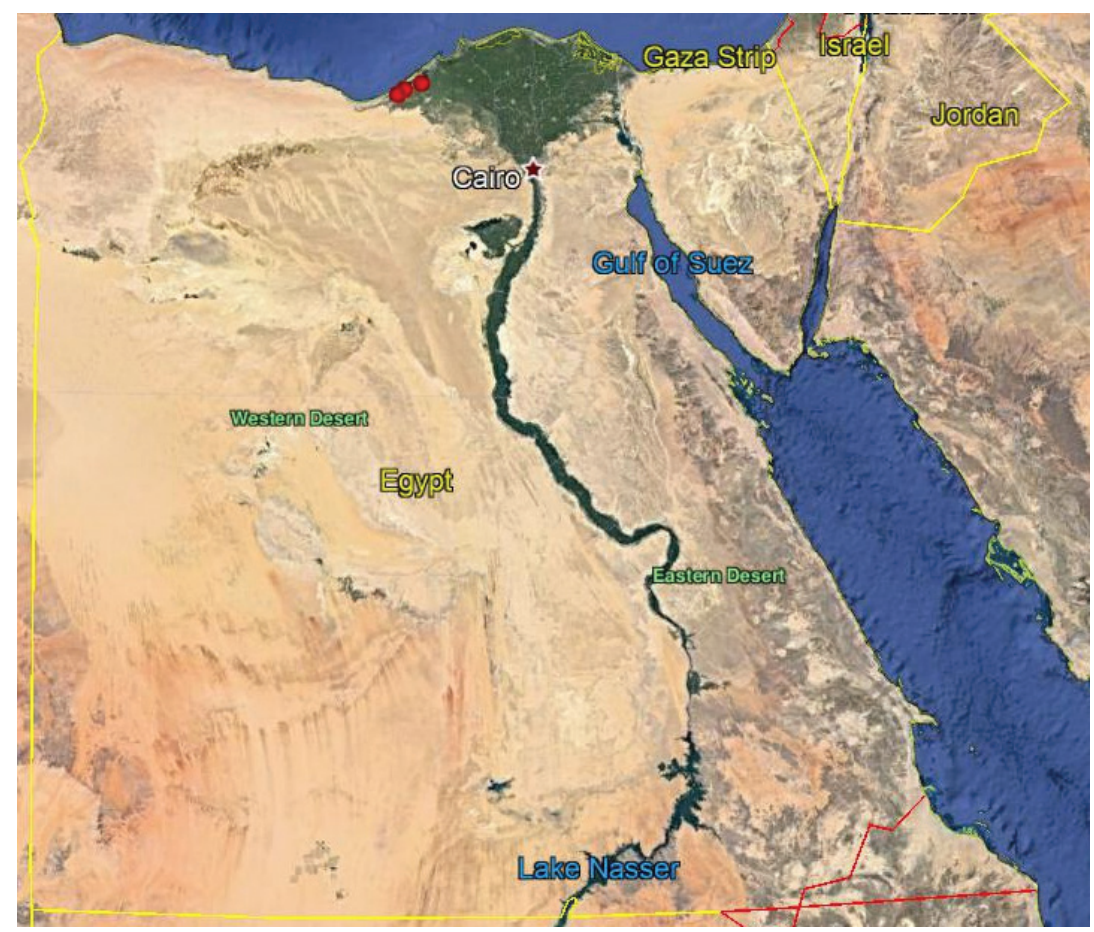

Map 5. Distribution of the species N. fasciatus in Egypt.

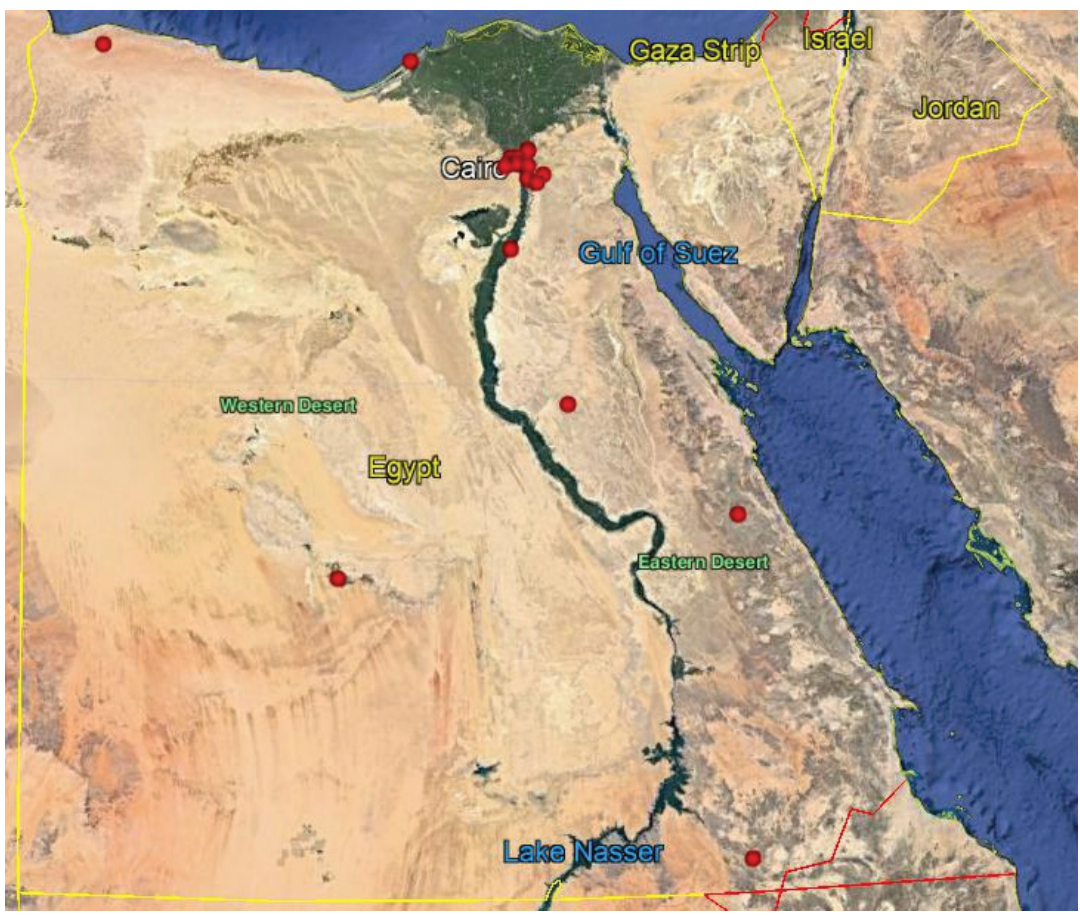

Map 6. Distribution of the species N. rufipes in Egypt. 
Furthermore, N. jullieni, a subspecies designated by Steyskal and El-Bialy (1967), is confirmed as a synonym of $N$. aegyptiacus.

The first species (N. caucasicus) does not occur in Egypt according to Sack (1933), Bequaert (1938), Bernardi (1973), Richter (1988), Narchuk (2007), or Kocak and Kemal (2013), whereas Paramonov (1951) reported it from North Africa but without examining any Egyptian material. It is also listed by Steyskal and El-Bialy (1967) citing the literature but not represented in the Egyptian reference collections.

The second species ( $N$. pallipes) is not represented in Egyptian collections. This species was previously considered to be an Egyptian species based on an erroneous interpretation of its type locality (Java) by Bequaert (1932), Bernardi (1973), and Richter (1988), for reasons unknown. The type locality was given by Olivier (1811) in his original description as Java, while describing seven new species from Egypt, although two of them were from Arabia and around the Caspian Sea. Olivier (1811) based his paper on material collected from different areas in the Middle East and his "Java" evidently refers to a place near Tel-Aviv in Israel currently known as Jaffa, not an Egyptian locality. We communicated with managers in Tel Aviv museum (Steinhardt museum), Diptera collection (Dr. Elizabeth Morguilis and Dr. Ariel-Leib Frieman), who checked the nemestrinid group and they do not have any specimens of this species in their collections. This species is also not mentioned in the list of Steyskal and El-Bialy (1967). Although it is believed that this species does not occur in Egypt, it may yet be found and recorded from the country.

The third species (N. persicus) is reported in Sack (1933) and Paramonov (1945) as an Egyptian species, but without any listing of Egyptian material. It is also mentioned as an Egyptian species by Steyskal and El-Bialy (1967) but is not represented in Egyptian reference collections; however, Bequaert (1932), Bernardi (1973), and Richter (1988) excluded it as an Egyptian species. The type locality in the original description was given as Iran by Lichtwardt (1909) and, consequently, this species is excluded from the Egyptian fauna.

Nemestrinus reticulatus is stated here as not having any specimens in Egyptian collections and is not excluded from the Egyptian fauna in our study because we trust the descriptions of Latreille (1809) who originally reported the species in Egypt. Our drawings of this species are reproduced from Bequaert (1938), Sack (1933), and Seguy (1926).

As we observed on the maps there are similarities in the distributions of $N$. aegyptiacus and $N$. rufipes, which are longitudinally scattered from Lower to Upper Egypt and the western and eastern deserts, while $N$. faciatus is concentrated only in some localities on the coastal strip in Alexandria. Nemestrinus exalbidus is dispersed around the lower Egyptian delta and a few localities in the western and eastern deserts. Nemestrinus ater has a crosswise distribution in the northern area of Egypt including Sinai, and the lower and upper Nile valley (see Map 1), and one record in the eastern desert, in addition to one locality near Libya.

The species of Nemestrinus are concentrated in the semiarid areas around the Nile delta, especially around Lower Egypt and in some arid areas in western, eastern, and the Egyptian Sinai deserts. The wider geographical distribution of Nemestrinus in the 
adjacent countries includes North Africa (Algeria, Libya, Morocco, and Tunisia) which all have a large percentage of the arid deserts that these nemestrinid species prefer. And at nearly the same latitude are Israel, Saudi Arabia, and Syria which also have arid areas (deserts) and may support species.

We found based that the seasonal imago flight activity of all Nemestrinus species in Egypt is in the spring season (March, April, and May) and only the species N. ater and N. exalbidus may also be activate in February.

\section{Acknowledgements}

We extend our sincere thanks to Dr. Neveen S. Gadallah, Dr. Magdy Shaban, and Dr. Yusuf Edmardash, the curators of the Cairo University Collection (CUC), Entomology Department, Faculty of Science, Cairo University. Deepest appreciation is also offered to Dr. Stefan Ober, Red Sea Environmental Centre (RSEC), and we also thank Dr. Bradley J. Sinclair, Canadian National Collection of Insects and Canadian Food Inspection Agency, Ottawa Plant Laboratory - Entomology, for kindly providing some valuable literature. Deepest thanks to Dr. Peter Sehnal, Naturhistorisches Museum Wien, Austria, for providing pictures of $N$. aegyptiacus specimen. Also deepest appreciation to Ms. Elena Grigoryeva, Mr. Sven Marotzke, and Bernhard Schurian, museum für Naturkunde, Germany, Berlin, who kindly provided pictures of the types (Nemestrinus aegyptiacus and $N$. rufipes and its synonym type $N$. lateralis) in different positions. Special thanks to Dr. Torsten Dikow, Smithsonian NMNH (USNM) research entomologist for Diptera, for identifying some Egyptian nemestrinid species existing in USNM. Many thanks to Dr. Elizabeth Morgulis, Dr. Netta Dorchin, and Dr. Ariel-Leib Frieman for checking the nemestrinid specimens in Tel Aviv museum (Steinhardt museum). Also, we are grateful to Dr. Adrian Pont (Oxford University Museum of Natural History, UK), who checked the English and made some corrections to this manuscript.

\section{References}

Bequaert J (1932) The Nemestrinidae (Diptera) in the V.v. Röder collection. Zoologischer Anzeiger 100: 13-33.

Bequaert J (1938) Sur quelques Nemestrinides palaearctiques, particulièrement d l'Iran. Annales de la Société entomologique de Belgique 78: 292-310.

Bernardi N (1973) The genera of the Family Nemestrinidae (Diptera: Brachycera). Arquivos de Zoologia 24(4): 211-318. https://doi.org/10.11606/issn.2176-7793.v24i4p211-318

Bigot J (1888) Enumeration des diptères recueillis en Tunisie dans la Mission de 1884 par M. Valery Mayet. Exploration scientifique de la Tunisie, Zoologie, Diptères. Imprimerie nationale, Paris, 11 pp. https://doi.org/10.5962/bhl.title.53767

Bischof J (1905) Neuropteren und Dipteren. In: Penther A, Zederbauer E (Eds) Ergebnisse einer naturwissenschaftlichen Reise zum Erdschias-Dagh (Kleinasien). Annalen des Naturhistorischen Hofmuseums, Wien 20: 170-179. 
Blanchard CE (1845) Histoire des insectes, traitant de leurs moeurs et leurs métamorphoses en général et comprenant une nouvelle classification fondée sur leurs rapports naturels. Coléoptères, orthoptères, thysanoptères, névroptères, lépidoptères, hémiptères, aphaniptères, strepsiptères, diptères, anoplures et thysanures 2: e20. https://doi.org/10.5962/bhl.title.35820

Efflatoun HC (1925) A new species of Nemestrina (Dipt. Nemestrinidae) from Egypt. Bulletin de la Société Entomologique d'Egypte 8: 357-360.

ElHawagry M, Gilbert F (2014) Zoogeographical affinities and faunal relationships of bee flies (Diptera: Bombyliidae) in Egypt. Zoology in the Middle East 60(1): 50-56. https://doi.or g/10.1080/09397140.2014.892339

Cumming JM, Wood DM (2017) Manual of Afrotropical Diptera. In: Diversity of Life, South African National Biodiversity Institute 1: 89-133.

Fischer G (1806) Observation d'un nouveau genre d'une nouvelle famille de diptères du Caucase. Mémoires de la Société impériale des naturalistes de Moscou 1: 217-227.

Fischer G (1812) Observation d'un nouveau genre de diptéres. Lue a la séance du 17 janvier 1806, avec des additions de l'année 1811. Mémoires de la Société impériale des naturalistes de Moscou 1(2): 184-198.

Koçak AÖ, Kemal M (2013) Diptera of Turkey. Priamus Supplement, Ankara, 411pp.

Latreille PA (1802) Histoire naturelle, genérale et particulière, des crustacés et des insectes. Ouvrage faisant suite à l'histoire naturelle générale et particulière, composée par Leclerc de Buffon, et rédigée par C.S. Sonnini, membre de plusieurs sociétés savantes. Tome Troisième. F. Dufart, Paris, 467. https://doi.org/10.5962/bhl.title.15764

Latreille PA (1809) Genera crustaceorum et insectorum secundum ordinem naturalem in familias disposita, iconibus exemplisque plurimis explicata. Tomus quartus et ultimus. A. Koenig, Parisiis and Argentorati [= Paris \& Strasbourg], 399 pp.

Lichtwardt B (1907) Uber die Dipteren-Gattung Nemestrina Latr. Zeitschrift für systematische hymenopterologie und dipterologie 7: 433-451.

Lichtwardt B (1909) Beitrag zur Kenntniss der Nemestriniden. Deutsche entomologische Zeitschrift 1: 113-127. https://doi.org/10.1002/mmnd.48019090117

Lichtwardt B (1919) Die Nemestriniden des Ungarischen National Museums in Budapest. Annales historico-naturales Musei nationalis hungarici 17: 24-27.

Macquart J (1840) Diptères exotiques nouveaux ou peu connus. Tome deuxième (1). Mémoires de la Société royale des sciences, de l'agriculture et des arts de Lille, Paris, 135 pp.

Teskey HJ (1981) Nemestrinidae. In: McAlpine JF, Peterson BV, Shewell GE, Teskey HJ, Vockeroth JR, Wood DM (Eds) Manual of Nearctic Diptera. Research Branch, Agriculture Canada Monograph, 585-588.

Narchuk EP (2007) Nemestrinid flies (Diptera, Nemestrinidae) in the Fauna of Eastern Europe and the Caucasus. Entomological Review 87(8): 1076-1085. https://doi.org/10.1134/ S0013873807080143

Olivier GA (1811) Insectes [i. e., Arthropoda), Pt.5]. Société de Gens de Savans et d'Artistes, Encyclopédie méthodique, Histoire Naturelle 8: 170-172.

Papavero N, Bernardi N (2009) Manual of Neotropical Diptera, Nemestrinidae. In: Depto. de Biologia - FFCLRP Universidade de São Paulo Ribeirão Preto, SP, Brazil 8: 1-11.

Paramonov SJ (1945) Bestimmungstabelle der Palaearktischen Nemestrinus-Arten (Nemestrinidae: Diptera). Revista Española de Entomología 21(3/4): 279-295. 
Paramonov SJ (1951) Bestimmungstabelle der palaearktischen Arten der Gattung Rhynchocephalus (Nemestrinidae: Diptera). Zoologischer Anzeiger 146(5-6): 18-127.

Pape T, Blagoderov B, Mostovski BM (2011) Order Diptera Linnaeus, 1758. In: Zhang Z-Q (Ed.) Animal biodiversity: An outline of higher-level classification and survey of taxonomic richness. Zootaxa, Magnolia Press, Auckland 3148: 223-237. https://doi.org/10.11646/ zootaxa.3148.1.42

Richter VA (1988) Family Nemestrinidae. In: Soós, Á. \& Papp, L. (eds.), Catalogue of Palaearctic Diptera 5: 171-181.

Richter VA (1997) Contributions to a Manual of Palaearctic Diptera. Vol. 2. Family Nemestrinidae. In: Science Herald, Budapest, 459-468.

Richter VA, Ovtshinnikova OG (1996) On the structure of male and female genitalia in Palaearctic nemestrinids (Diptera, Nemestrinidae). International Journal of Dipterological Research 7(4): 241-249.

Rondani C (1850) Osservazioni sopra alquante specie di esapodi ditteri del Museo Torinese. Nuovi Annali delle Scienze Naturali e Rendiconto delle Sessioni della Società Agraria e dell'Accademia delle Scienze dell'Istituto di Bologna 2(3): 165-197.

Sack P (1933) Die Fliegen der palaearktischen Region, Nemestrinidae. In: Lindner E (Ed.) Stuttgart. Vol. 4 (22): 1-42.

Steyskal GC, El-Bialy S (1967) A list of Egyptian Diptera with a bibliography and key to families. Ministry of Agriculture Technical Bulletin 3: e37.

Thompson FC, Pape T (2021) Systema Dipterorum. http://www.diptera.org/ [accessed August 2021]

Villeneuve J (1912) Notes synonymiques. Wiener Entomologische Zeitung 31: 1-97.

Wiedemann CRW (1828) Aussereuropaische zweiflugelige Insekten. Zuerst theil. Schulz, Hamm, 608 pp. 